\title{
Dental Materials
}

\section{High Phosphate Content in Bioactive Glasses Promotes Osteogenesis in vitro and in vivo \\ --Manuscript Draft--}

\begin{tabular}{|c|c|}
\hline Manuscript Number: & DENTMA-D-20-00341R1 \\
\hline Article Type: & Full Length Article \\
\hline Keywords: & $\begin{array}{l}\text { Bioactive glass; phosphate content; Apatite formation; controlled release; Cell } \\
\text { Differentiation; osteogenesis }\end{array}$ \\
\hline Corresponding Author: & $\begin{array}{l}\text { Xiaojing Chen } \\
\text { Central South University Xiangya Stomatological Hospital } \\
\text { Changsha, CHINA }\end{array}$ \\
\hline First Author: & Yiping Li \\
\hline \multirow[t]{9}{*}{ Order of Authors: } & Yiping Li \\
\hline & Lu Chen \\
\hline & Xiaohui Chen \\
\hline & Robert Hill \\
\hline & Songyou Zou \\
\hline & Minyuan Wang \\
\hline & Yuting Liu \\
\hline & Jue Wang \\
\hline & Xiaojing Chen \\
\hline Abstract: & $\begin{array}{l}\text { Objectives. High phosphate content was found to significantly increase apatite } \\
\text { formation of bioactive glasses (BGs) in vitro. However, there is very limited } \\
\text { understanding of the effect of phosphate contents on osteogenesis which is important } \\
\text { for clinical applications. The aims of this study were to investigate how phosphate } \\
\text { content influences apatite formation ability of bioactive glasses in a -MEM culture } \\
\text { medium and whether high phosphate content in bioactive glasses promotes } \\
\text { osteogenesis in vitro and in vivo. } \\
\text { Methods. Four phosphate containing bioactive glasses were synthesized via a melt- } \\
\text { quench method and characterized using X-ray powder Diffraction (XRD), TGA-DSC } \\
\text { and Fourier transform infra-red spectroscopy (FTIR). The apatite formation ability in a- } \\
\text { MEM culture medium and the in vitroand in vivo osteogenic potential of these bioactive } \\
\text { glass were explored. } \\
\text { Results. FTIR spectra confirmed faster apatite formation with an increase in phosphate } \\
\text { content. The culture media containing ions released from the BGs showed enhanced } \\
\text { cell viability and alkaline phosphatase activity of osteoblasts. Osteoblasts cultured with } \\
\text { extracted BGs culture media generally showed increased proliferation, mineralized } \\
\text { nodule formation, osteogenic and angiogenic genes expression with an increase in } \\
\text { phosphate content in the glass compositions. An in vivo study demonstrated a larger } \\
\text { amount of new bone formation in the calvarial defects implanted with high phosphate } \\
\text { containing BG granules compared with that of BG without the presence of phosphate } \\
\text { at } 8 \text { weeks post-surgery. } \\
\text { Significant. The presence of higher phosphate content accelerates apatite formation } \\
\text { and promotes osteogenesis, indicatingthat both apatite formation and osteogenesisof } \\
\text { bioactive glasses can be tailored by varying phosphate content for specific clinical } \\
\text { needs and personalized treatments. }\end{array}$ \\
\hline Response to Reviewers: & $\begin{array}{l}\text { We thank three reviewers and editor for appreciating the interest of the paper and the } \\
\text { constructive comments provided. A word file of Respond to Reviewers has been } \\
\text { attached in the "Attach Files" section. }\end{array}$ \\
\hline
\end{tabular}




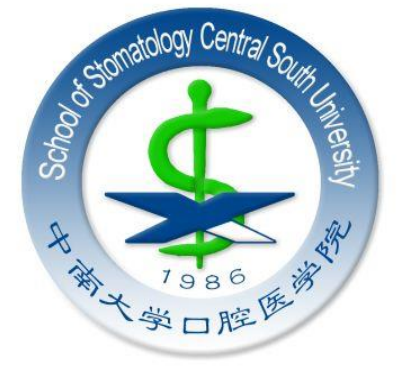

\author{
Dr. Xiaojing Chen \\ Xiangya School of Stomatology \\ Central South University \\ 72 Xiangya Rd, Kaifu District, \\ Changsha, Hunan \\ 410078 \\ Email: xiaojing.chen@csu.edu.cn
}

Tel: 008618569086831

$29^{\text {th }}$ Oct 2020

To the Editors of Dental Materials

Dear Prof. Watts,

RE: Manuscript Number: DENTMA-D-20-00341, Article Title: "High Phosphate Content in Bioactive Glasses Promotes Osteogenesis in vitro and in vivo".

We would like to thank you and the reviewers for their constructive comments and suggestions. We have revised our manuscript based on the reviewers' comments. Details of our amendments to the manuscript and responses are elaborated in the enclosed revised manuscript and response to reviewers.

Thank you very much again for considering this article for publication in Dental Materials.

Yours faithfully,

Dr. Xiaojing Chen

(on behalf of all co-authors) 
Manuscript Number: DENTMA-D-20-00341

Response to General Comments: We thank three reviewers for appreciating the interest of the paper and the constructive comments provided.

\section{Response to Reviewer \#1:}

1) The authors used bioactive glass with $38 \mu \mathrm{m}$ in particle size for in vitro study and 63-90 $\mu \mathrm{m}$ for in vivo study (page 5, line 12-13). The authors are advised to mention the reason why the different sized particles were applied in these studies.

Bioactive glass with a particle size less than $38 \mu \mathrm{m}$ is commonly used in vitro to study bioactive glass dissolution and apatite formation; and was recommended by Technical Committee 4 (TC04) of the International Commission on Glass (ICG). The same particles size range was previously used in my research group and has therefore been used in the present study not only complies with recommendation but also allows comparison with previous results.

The bioactive glasses with high phosphate content investigated in the present study have been designed as an alternative bone grafting substitute for 45S5 granules. When used in vivo, if the particle size is too small, rapid glass degradation will not allow sufficient time for new bone formation, in contrast, large glass particles with significantly reduced surface areas are more appropriate for a balanced glass degradation and new bone formation. The particle size of 63-90 $\mu \mathrm{m}$ was selected for the present study based on our preliminary results.

As suggested, the reason why two different sized particles were applied have been included materials and methods (P5).

2) The authors explained wavelength of FT-IR between $600 \mathrm{~cm}-1$ and $560 \mathrm{~cm}-1$ (page 5, line 26), however I cannot find this range in Fig. 1c. I recommend the authors to fix this issue.

We thank reviewer for the comment and have revised Fig. 1c (P22) to make the wavelength range clear for reader.

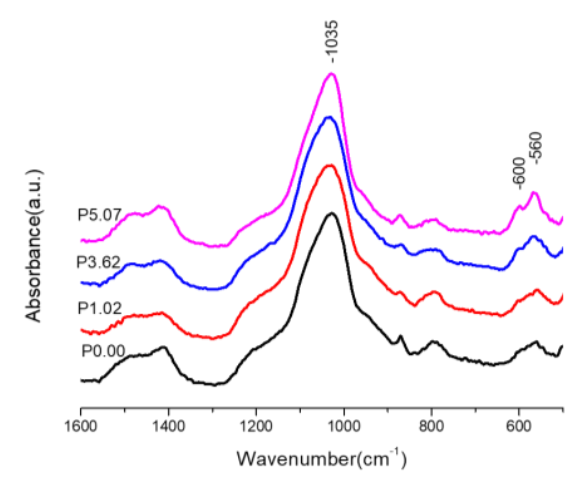

3) Figure 2; ICP data should be analyzed statistically and described in details in this caption. 
For ICP experiment, 2 separate samples from each immersion point of interest of all studied glasses were prepared and measurements were repeated in triplicates, the Mean \pm SD of the 6 measurements were presented as measured ion concentration for the glass at the immersion point of interest. Statistical analysis of the collected ICP data between sample groups has been conducted using ANOVA with a significance level of 0.05 .

Due to the large number of figures and tables to be presented, we did not include a table listing the results and the significant difference between results, but plotted Mean and SD against immersion time as ion release profile in Fig 2 to share the overall trend. The SD for sample group presented as error bar is relatively small and overlaps with the symbol used for data point on graph.

As suggested, we have included how the statistical analysis of the ICP data was completed in methods and also added "Note that where error bars are not seen, they are smaller than the data point." in the figure caption (P23).

4) Figure $3 \mathrm{~b}$ and to this part; the authors mentioned that bioactive glass with high phosphate content (5.07 mol\%) accelerated cell growth (page 9, line 30-32). But given what I have seen Fig. 3a, cell proliferation with P5.07 and P0.00 showed no significant difference on day 3 and 7 . The authors should resolve this contradiction.

It is true that cell proliferation with P5.07 and P0.00 showed no significant difference on day 3 , however, a clear difference was noticed on day 7 . We have revised page 9, line 30-32 to better explain the results shown in Fig.3.

5) Figure 5 and 6; the authors set the all control values as 1 , but these data should be split into each day, otherwise these data may mislead readers to see that gene/protein expressions in control are stable during the observation periods. In a sense, we cannot compare the expression levels among the samples with different culturing days. And, what is the P1 group described in captions?

We thank reviewer for the valuable comment. We did set the expression of control at different culturing day as 1 , and compared the expression level of the BGscontained culture media and control at the same culturing days. We have revised the experimental method session (P7) and figure captions for Figure 5 and 6 (P19, $\mathrm{P} 26$ \& 27).

P1 was referring to P0.00 glass without phosphate. When fabricating/making the glasses, we named glasses as P1, P2, P5, but have decided to designate glass according to Phosphate content, e.g. P0.00, and may have missed this particular one. We have checked throughout the manuscript, and updated P1 to P0.00.

6) Figure 7; the immunofluorescence images have to be shown with same magnification. The authors are advised to replace the images of day 7 (Fig. 7a), because it is very hard to discriminate the osteopontin located within nucleus by such low magnification.

We thank reviewer for the comment and have replaced the image for Figure $7 \mathrm{a}$ and update the figure caption (P28). 
A

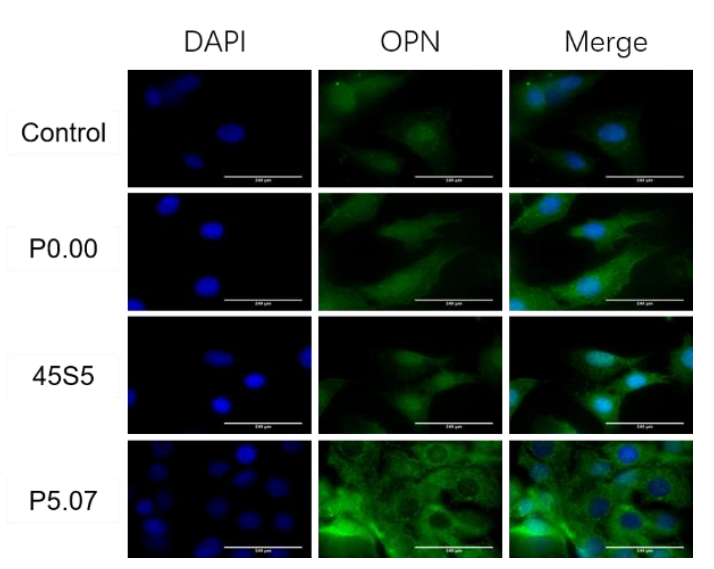

b

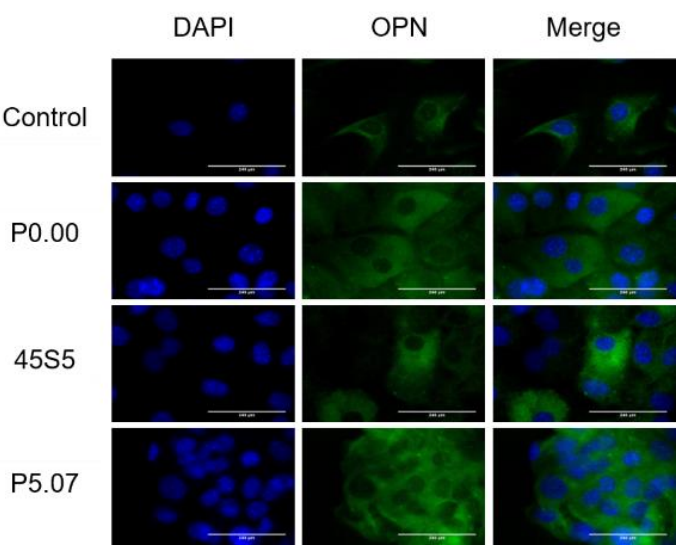

Fig 7. Representative fluorescence images show staining of DAPI (blue) and OPN (green) in MC3T3-E1 cells following BGs conditioned culture media treatment for (a) $7 \mathrm{~d}$ and (b) $14 \mathrm{~d}$. Scale bar $240 \mu \mathrm{m}$.

7) Figure 8 and to this part; bioactive glass tested could be appeared in micro-CT or biodegraded by 8 weeks? Without histological evaluation, we cannot prevent the possibility that remained materials were captured by micro-CT. The authors should provide the histological images or adequate perception in discussion section.

Discussion of potential material been captured by micro-CT has been included in discussion, the importance of conducting histological evaluation has also been highlighted.

We are currently applying for funding to pursue the histological evaluation and will conduct this as a separate study.

\section{Response to Reviewer \#2:}

1) Proof reading is required, such as Bone loss and defects caused by oral diseases, for instant periodontitis, peri-implantitis, and maxillofacial defects are very common clinically and reduce the quality of life for patients.

We have proof read the manuscript and revised Grammar throughout.

2) In order to better inform and predict the clinical success of soda-lime-phosphosilicate BGs, understand their influence on osteogenesis, how well do they perform in animal models, and the correlation between the in vitro and in vivo results are vital.

We totally agree with reviewer and this is why we conducted the presented study.

3) Glass transition temperature ( $\mathrm{Tg}$ ) of those glasses was reported by O'Donnell et al. [10], what is the reason to repeat it in this study? 
The glass compositions investigated in the present study were designed by O'Donnell and a co-author of this study. Although the furnaces used for the present study is the same model from what O'Donnell used, however, small difference in temperature calibration may exist and influence the glass composition. $\mathrm{T}_{\mathrm{g}}$ is an important parameter for glass and has a relationship with glass composition. The reason why we repeated $\mathrm{T}_{\mathrm{g}}$ measurements was to compare with the results reported by O'Donnell et al. [10] so at to confirm that what we synthesized are the same as what O'Donnell achieved so that the results obtained on this work can be compared with the in vitro study carried out previously by O'Donnell.

4) Why the $4 \mathrm{~h}$ BG conditioned $\alpha$-MEM was chosen for cell study? How about the behaviors of 12, 24 and $72 \mathrm{~h}$ medium? In the $4 \mathrm{~h}$ conditioned medium, the released $\mathrm{Ca}$ and $\mathrm{Si}$ concentrations significantly increased, especially the $\mathrm{pH}$ reached to 8, which seems cytotoxic to cell growth

Yes, compare with control, there was an increase in $\mathrm{Ca}$ and $\mathrm{Si}$ in the $4 \mathrm{~h}$ conditioned medium, suggesting that bioactive glass dissolved and released ions. A reduction on ion concentration was observed with an increase in BG immersion time. The time point that shows the highest ion and $\mathrm{pH}$ level ( $4 \mathrm{~h}$ in this case) represents the highest cytotoxicity risk to cell growth, therefore, it was of great interest.

Actually, the cytotoxicity experiments were conducted using 4, 12, 24 and $72 \mathrm{~h} \mathrm{BG}$ conditioned $\alpha$-MEM for 1, 3 and $7 \mathrm{~d}$ experiment. Similar trends were seen, generally, the cell viability for BG conditioned $\alpha$-MEM were higher than that for $\alpha$-MEM control, indicated that the BGs conditioned $\alpha$-MEM with different treatment time are not cytotoxic to cells.

The results are shown here:

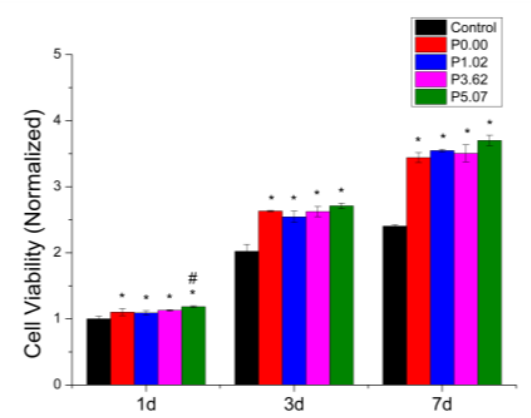

A

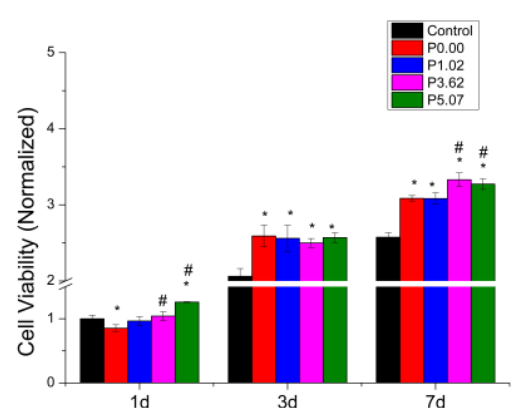

b 


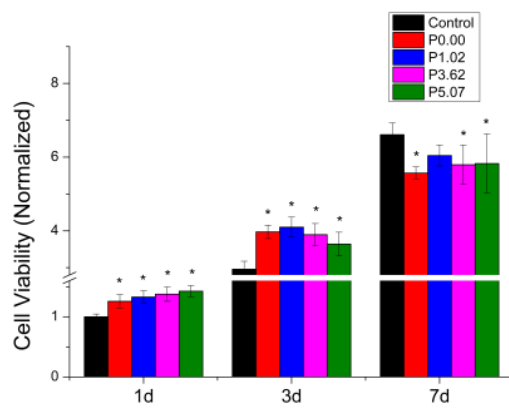

$\mathrm{c}$

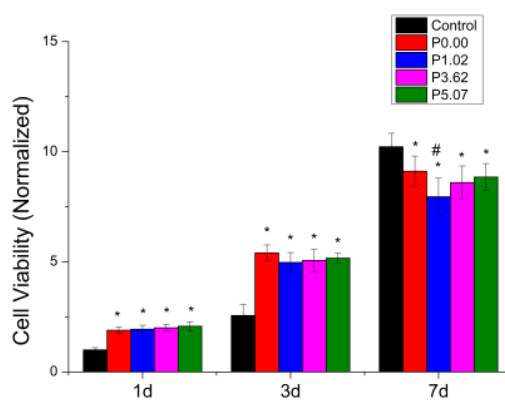

d

Figure. Cell viability of different BG-conditioned $\alpha$-MEM on MC3T3-E1 a) 4h, b)

$12 \mathrm{~h}, \mathrm{c}) 24 \mathrm{~h}$ and d) $72 \mathrm{~h}$, data are presented as the Mean $\pm \mathrm{SD}(\mathrm{n}=6), * \mathrm{P}<0.05$, compared with the control group. \#P $<0.05$, compared with glass $\mathrm{P} 0.00$.

5) Cell Counting Kit-8 assay is more to measure cell viability/proliferation, instead of metabolic activity. Cell metabolic analysis includes such as oxygen consumption, glycolysis, and fatty acid metabolism.

6) Please add more details on the alizarin red s staining extraction

7) Please double check the reference format and numbering

8) Please add the antibody information used in western blot and IF, especially the dilution ratios

9) Please add more details in BV/TV analysis, such as how to define the new bone, how to calculate the bone volume and were BG granules visualized in microCT scanning.

We thank the reviewer for comment 5-9, relevant amendments on manuscript have been made according to the suggestions.

10) Apatite formation, only $72 \mathrm{~h} \mathrm{BG}$ was tested, how about the results of 4,12 and $24 \mathrm{~h}$ immersion in a-MEM? How about the apatite confirmation by XRD?

We have looked at apatite formation at all different immersion in a-MEM $(4,12$, 24 and $72 \mathrm{~h}$ ). A decrease in the intensity of non-bridging oxygen bond at $920 \mathrm{~cm}^{-1}$, and the more significant appearance of the characteristic split speaks at 600 and 560 $\mathrm{cm}^{-1}$ were found with increasing immersion time from 4 to $72 \mathrm{~h}$. Due to the number of figures to be included, we have decided not to include all FTIR spectra.

We did conduct XRD measurement on all glass powders retrieved after immersion and noticed very broad humps on the amorphous halo - as a result of diffraction peak broadening due to fine crystals. We did not include all XRD patterns, as the main focus of the present manuscript is whether high phosphate content in bioactive glass promotes osteogeneis in vitro and in vivo.

11) Fig 6, please include representative bands of Western blot, such as the $7 d$ control VEGF was significant greater than the experimental groups, which was not consistent with the quantitative analysis 
Three paralleled western blot experiments were carried, due to the number of figures to be included, only one representative image of VEGF was shown here, while the result of quantitative analysis was exhibited as mean \pm SD in Fig 6 b. Please find the additional representative bands of WB as following:

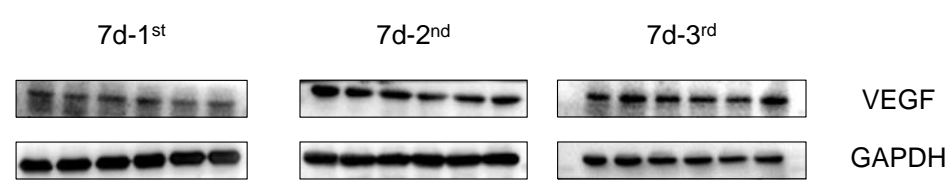

12) Fig7, magnification of IF images should be kept the same, especially the $7 d$ images with a low magnification, which was not clear enough to indicate OPN protein was located in both extracellular matrix (ECM) and nucleus

Thank you, this was also pointed out by reviewer 1, and has been addressed (P28).

13) What is the rational of comparing P0 and P5.07 in vivo, instead of such as 45S5 vs P5.07, which will be more efficient to demonstrate the osteogenesis effect of high phosphate.

The 4 glasses investigated in the present study have a constant network connectivity and a gradual increase in Phosphate content, which allow us to better understand the relationship between Phosphate content and glass dissolution, apatite formation etc. In vitro results suggested that high phosphate content promotes osteogenesis, therefore, the phosphate free composition investigated was selected alongside the highest Phosphate contained glasses for a pilot in vivo study.

45S5 has a phosphate content at $2.6 \mathrm{~mol} \%$ and a $\mathrm{NC}$ of 2.12, which have 2 key variables compared with the glass compositions studied, which make direct comparison challenging. We do agree with reviewer that it is worth comparing with $45 \mathrm{~S} 5$, and indeed conducted some experiment - will run as a separate small project.

14) Why 45S5 was only included in western blot and IF?

Western blot and IF were completed by another student who have included 45S5. Although 45S5 was not the focus of the present study and has slightly higher NC (2.12), the results provided additional information, was therefore kept and presented.

\section{Response to Reviewer \#3:}

We thank reviewer for the comment on MC3T3-E1 cells which has been added to method.

As I read it, the conditioned media was prepared in neat alpha-MEM - at what point were the supplements (P/S, FBS) added? The text does not make reference to when that happens. It raises some other questions, if prepared in neat alpha-MEM, what would be the release/consumption kinetics if prepared in complete (ie plus P/S, FBS) medium? 
Very good comment and questions on conditioned media preparation and release kinetics. We have revised methods and made how condition media was prepared clear for readers (P6).

2.4.2. The CCK-8 test infers cell number is based on there being no disturbances on activities of the intracellular dehydrogenases in the different conditions. Could the validity of these results be backed up with DNA content/well as an additional proof of increased proliferation?

This is a great question certainly worth pursuing, however, we have not yet attempted to quantify DNA content, but have revised and mentioned this in discussions and future work (P13).

3.2 Have the background levels of Ca, P, Si been subtracted from the conditioned media experiments? This might give a clearer result as to the release/consumption kinetics of each ion species. Why does the BG with the highest Si content have the lowest release (Fig 2c). (Is it convention to start the axis of graph at zero (fig 2a,d)?).

The background level has not been subtracted. As the measured ion concentration is the overall ion in solution as a result of the ion available in media, ion release from glass and consumption of ions to form apatite or other precipitation. If subtracted, we could have a negative ion concentration which may not be as straightforward for readers, therefore, we have decided to keep the raw measurement results. However, I have revised the method session and the graph (inserting a baseline ion concentration as reference). An additional line in figure caption have also been added to increase clarity.

Despite the NC of all investigated glasses is constant, the increase in phosphate content alongside with $\mathrm{Ca}$ and $\mathrm{Na}$ may have resulted in a less "packed" glasses that degraded faster with higher ion release. Therefore, P0.00 with the lowest phosphate content is expected to have the lowest release.

It is convention to start the axis of graph at zero (as Fig $2 \mathrm{~b}$ and c), the intersect for the y axis for Fig 2.a and Fig $2 \mathrm{~d}$ has not been set at zero so as to void squashing the results together and allow emphasis of the results. We have tried to include a break for $y$ axis, however, the way how Fig 2.a and Fig 2d still look better.

Fig 8 - Is the graph correct? The image would infer that the difference between the two preparations is much greater.

We thank the reviewer's comment. The graph presented was the best to our knowledge. The individual difference of SD rats that used in the pilot in vivo study, was likely lead to a clear variation in micro-CT results. A systematic in vivo study with larger sample numbers will be conducted in the near future might reduce the difference within the group.

All the data suggests phosphate is having an effect to promote osteogenesis, however, there are not any truly stand out data sets, and intimates only small cellular differences might still be beneficial in promoting changes in skeletal bone mass in the skull. The effect of these materials on alveolar bone would be most interesting. 
This is exactly the reason why we conducted the present study and tried to establish whether in vitro results provide solid evidence to indicate the success for in vivo application. The in vivo study was a pilot study testing feasibility, what we can conclude is limited. We did recognize the points that reviewer raised and agree that the effect of these high phosphate bioactive glasses on alveolar bone is interesting and worth investigating. However, all data collected indeed suggested phosphate have an effect to promote osteogenesis. We think it is fair to share and report the results with the community and simulate discussions for future work. We have revised discussions, and included the suggestions made. 
High Phosphate Content in Bioactive Glasses Promotes Osteogenesis in vitro and in vivo

Yiping Li ${ }^{\mathrm{a}}$, Lu Chen ${ }^{\mathrm{a}}$, Xiaohui Chen ${ }^{\mathrm{b}}$, Robert Hill' ${ }^{\mathrm{c}}$, Songyou Zou ${ }^{\mathrm{a}}$, Minyuan Wang ${ }^{\mathrm{a}}$, Yuting Liu ${ }^{\mathrm{a}}$, Jue Wang ${ }^{\mathrm{a}}$, Xiaojing Chen ${ }^{\mathrm{a}, \mathrm{c}^{*}}$

a.Hunan Key Laboratory of Oral Health Research \& Hunan 3D Printing Engineering Research Center of Oral Care \& Hunan Clinical Research Center of Oral Major Diseases and Oral Health \& Xiangya Stomatological Hospital \& Xiangya School of Stomatology, Central South University, Changsha, 410008, Hunan, China.

${ }^{\mathrm{b}}$ Division of Dentistry, School of Medical Sciences, The University of Manchester, Manchester, UK.

${ }^{c}$. Institute of Dentistry, Dental Physical Sciences Unit, Barts \& The London School of Medicine and Dentistry, Queen Mary University of London, UK

${ }^{*}$ Corresponding author

E-mail address: xiaojing.chen@csu.edu.cn (Xiaojing Chen)

Address: 72 Xiangya Road, Kaifu District, Changsha, Hunan Province, China, 410008

Tel: +86 (0731) 84800281 
DENTMA-D-20-00341, R1

\section{Highlights}

The effect of phosphate content in soda-lime-phospho-silicate bioactive glass on osteogenesis at cell and organism levels has been investigated for the first time.

Apatite formation and osteogenesis of bioactive glasses can be enhanced by increasing phosphate content.

The in vivo study demonstrated that high phosphate content BG (P5.07) enhanced bone formation in rat calvarial defect at $8 \mathrm{w}$ comparing to phosphate free BG (P0.00).

The findings demonstrate the potential of predicting in vitro and in vivo bone formation ability of bioactive glass based on in vitro investigations, such as the conventional bioactivity study using SBF. 


\section{Graphical abstract}

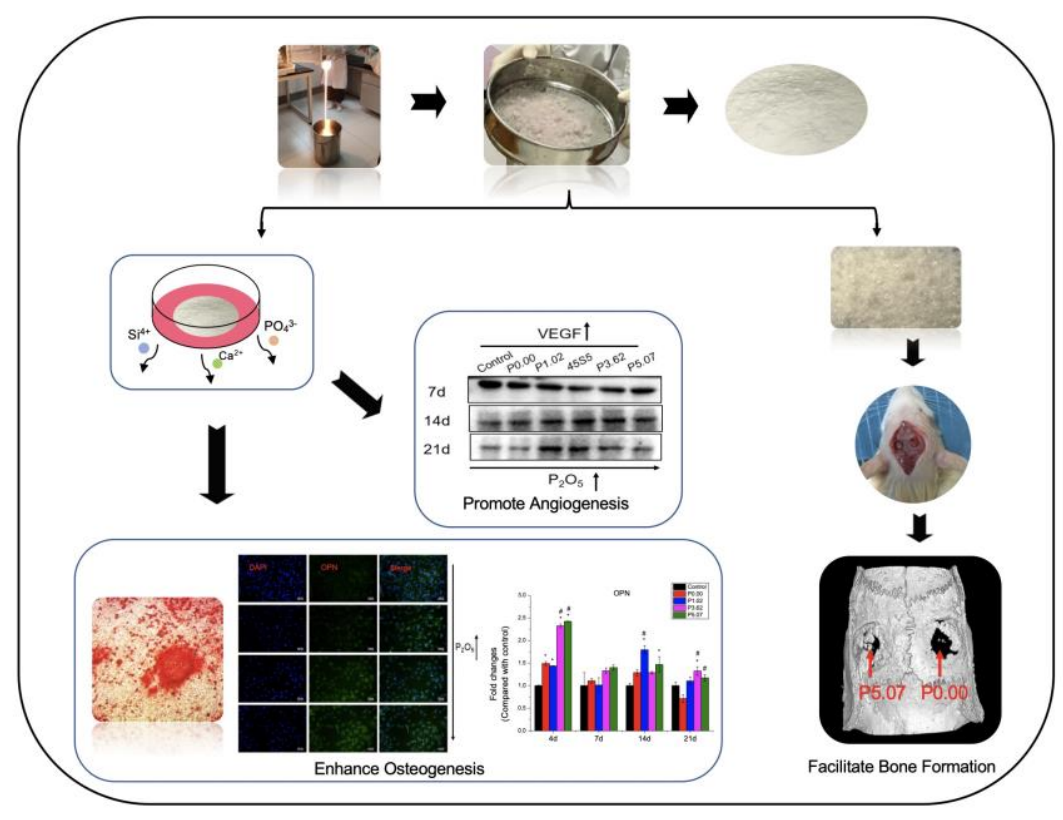


DENTMA-D-20-00341, R1

\begin{abstract}
Objectives. High phosphate content was found previously to significantly increase apatite formation of bioactive glasses (BGs) in vitro. However, there is very limited understanding of the effect of phosphate contents on osteogenesis which is important for clinical applications. The aims of this study were to investigate how phosphate content influences apatite formation ability of bioactive glasses in $\alpha$-MEM culture medium and whether high phosphate content in bioactive glasses promotes osteogenesis in vitro and in vivo.
\end{abstract}

Methods. Four phosphate containing bioactive glasses were synthesized via a meltquench method and characterized using X-ray powder Diffraction (XRD), TAGA-DSC and Fourier transform infra-red spectroscopy (FTIR). The apatite formation ability in $\alpha$-MEM culture medium and the in vitro and in vivo osteogenic potential of these bioactive glass were explored.

Results. FTIR spectra confirmed faster apatite formation with an increase in phosphate content. The culture media containing ions released from the BGs showed enhanced cell viability and alkaline phosphatase activity of osteoblasts. Osteoblasts cultured with extracted BGs culture media generally showed increased proliferation, mineralized nodule formation, osteogenic and angiogenic genes expression with an increase in phosphate content in the glass compositions. An in vivo study demonstrated a larger amount of new bone formation in the calvarial defects implanted with high phosphate containing BG granules compared with that of BG without the presence of phosphate at 8 weeks post-surgery.

Significant. The presence of higher phosphate content accelerates apatite formation and promotes osteogenesis, indicating that both apatite formation and osteogenesis of bioactive glasses can be tailored by varying the phosphate content for specific clinical needs and personalized treatments.

Key words: bioactive glass; phosphate content; apatite formation; controlled release; cell differentiation; osteogenesis. 


\section{Introduction}

Bone loss and defects caused by oral diseases, for instant periodontitis, peri-implantitis, and maxillofacial defects are very common clinically in clinic and reduce the quality of life for patients. Autologous and allogenic bone grafts are routinely applied as a treatment. However, these materials are associated with problems, such as insufficient source and immune rejection- [1-3]. Therefore, alternative synthetic biomaterials are required, with good biocompatibility and the ability to promote osteogenesis [4].

The first bioactive glass (BG), 45S5 Bioglass ${ }^{\circledR}$ was developed in 1969 by Hench [5] to fill voids in the damaged bone without adverse effect from human body. 45S5 Bioglass ${ }^{\circledR}$ comprises in mol\% $46.1 \mathrm{SiO}_{2}-24.4 \mathrm{Na}_{2} \mathrm{O}-26.9 \mathrm{CaO}-2.6 \mathrm{P}_{2} \mathrm{O}_{5}$, can degrade and lead to rapid release of ions (e.g., $\mathrm{Si}^{4+}, \mathrm{Ca}^{2+}, \mathrm{PO}_{4}{ }^{3-}$ and $\mathrm{Na}^{+}$) and the formation of a hydroxycarbonated apatite (HCA) layer when in contact with simulated body fluid (SBF) [6]. The released ions were reported to stimulate osteogenic genes associated with the differentiation of osteoblasts [7] and the formed HCA can bond to collagen fibrils and facilitate the development of a strong interface between the glass and the host bone [6].

Despite the fact that 45 S5 Bioglass ${ }^{\circledR}$ has been widely used in orthopedics and dentistry since 1985 [8], the apatite formation is relatively slow. Hill and his group designed new BGs with different phosphate contents based on 45S5 Bioglass ${ }^{\circledR}$ and found that high phosphate contents $(6.33 \mathrm{~mol} \%)$ in BG showed superior bioactivity in term of faster apatite formation in SBF in comparison to 45S5 Bioglass ${ }^{\circledR}$ (pPhosphate content of 2.6 $\mathrm{mol} \%$ ) [9]. It is believed that glass structure and composition have a strong influence on glass bioactivity, and an enhanced glass degradation can be achieved when a glass moves from a three-dimensional network to a more disrupted linear chain or end members structures [10]. Edén [11] proposed that bioactivity can be increased monotonically with an increase in phosphorus content of the bioactive glass providing phosphorus remains predominantly as orthophosphate and the network connectivity (NC) is less than 2.6. O'Donnell et al. [9] investigated the influence of phosphate content on the bioactivity of a $\mathrm{SiO}_{2}-\mathrm{Na}_{2} \mathrm{O}-\mathrm{CaO}-\mathrm{P}_{2} \mathrm{O}_{5}$ system with a constant glass network connectivity and demonstrated faster formation of HCA with an increase in the phosphate content.

In vitro degradation and apatite formation in SBF have been used as an indicator for the formation of an intimate bond to host bone in vivo, however, there is a lack of evidence on the reliability of estimating BG bioactivity at cytology, animal and human levels based on bioactivity studies in SBF. More importantly, there is no study on evaluating the effect of phosphate contents in BG on osteogenesis in vitro and in vivo yet.

In order to better inform and predict the clinical success of soda-lime-phospho-silicate BGs, understanding of their influence on osteogenesis, how well do they perform in animal models, and the correlation between the in vitro and in vivo results are vital. Here, it was hypothesized that high phosphate content in bioactive glasses promotes apatite formation in culture media and facilitates osteogenesis in vitro and in vivo. The 
influence of phosphate on glass bioactivity in $\alpha$-MEM was evaluated. More importantly, in vitro response of osteoblast cell MC3T3-E1 and in vivo response of calvarial defects to the bioactive glasses with different phosphate contents were investigated systematically.

\section{Materials and methods}

\subsection{Bioactive glass fabrication}

BGs in the system of $\mathrm{SiO}_{2}-\mathrm{P}_{2} \mathrm{O}_{5}-\mathrm{CaO}-\mathrm{Na}_{2} \mathrm{O}$ designed by O'Donnell and team [10] (Table 1) were synthesized by a melt-quench method. Briefly, a mixture of glass reagents was melted in a platinum/rhodium crucible at $1395^{\circ} \mathrm{C}$ for $1 \mathrm{~h}$ and quenched into water to prevent crystallization. The glass frits were dried, ground and sieved. The collected glass powders with a particle size less than $38 \mu \mathrm{m}$ was-were used for further bioactivity and in vitro studies according to [12] and the unified in vitro evaluation method for glass bioactivity recommended by Technical Committee 4 (TC04) of the International Commission on Glass (ICG) $[13]$ REF t; while the gGlass granules with a particle size between 63 and $90 \mu \mathrm{m}$ were applied for animal study based on our preliminary results as - 1 Larger glass granules with significantly reduced surface areas isare more appropriate for a balanced glass degradation, and apatite and new bone formation. -

\subsection{Bioactive glass characterization}

The amorphous structure of the glass was confirmed by X-ray Diffraction (XRD) using Philips X'Pert PRO theta-theta PW3050/60 diffractometer with PW3064 sample spinner and $\mathrm{X}^{\prime}$ Celerator $\left(2.122^{\circ}\right.$ active length) 1D-detector in Bragg-Brentano geometry employing a Copper Line Focus X-ray tube with $\mathrm{Ni} \mathrm{k} \beta$ absorber $(0.02 \mathrm{~mm}$; $\mathrm{K} \beta=1.392250 \AA) \mathrm{K} \alpha$ radiation $(\mathrm{K} \alpha 1=1.540598 \AA, \mathrm{K} \alpha 2=1.544426 \AA$, $\mathrm{K} \alpha$ ratio 0.5 , $\mathrm{K} \alpha \mathrm{av}=1.541874 \AA$ ). Data was collected at $5-70^{\circ} 2$ theta with a step size of $0.033^{\circ}$ and a step time of $200 \mathrm{~s} / \mathrm{step}$. The sample was mounted on a silicon low background holder. Fourier Transform Infrared Spectroscopy (FTIR, ALPHA II, Bruker UK) was also-used to collected spectra in the range of 500 to $1600 \mathrm{~cm}^{-1}$ of wavenumber at a resolution of $4 \mathrm{~cm}^{-1}$.

The glass transition temperature $\left(\mathrm{T}_{\mathrm{g}}\right)$ was determined using TGA-DSC (TGA/DSC 3+, Switzerland). Glass frit $(40 \mathrm{mg})$ was heated at a rate of $20^{\circ} \mathrm{C} / \mathrm{min}$ under nitrogen (a flowat a rate of $60 \mathrm{ml} / \mathrm{min}$ ) from 25 to $1100^{\circ} \mathrm{C}$ in a Pt crucible. Alumina was used as reference. $\mathrm{T}_{\mathrm{g}}$ was extracted from the DSC traces with an accuracy of $\pm 5^{\circ} \mathrm{C}$ and compared with data reported previously by O'Donnell et al. [10].

\subsection{Bioactivity Evaluation}

Glass powders (75 mg each) were dispersed in $50 \mathrm{~mL} \alpha$-MEM cell culture medium, corresponding to a concentration of $1.5 \mathrm{~g} / \mathrm{L}$ [1123-14]. All samples were placed in an orbital shaking incubator (IKA ${ }^{\circledR} \mathrm{KS} 4000 \mathrm{i}$ control, Germany) at $37^{\circ} \mathrm{C}$ with an agitation rate of $60 \mathrm{rpm}$ for $4,12,24$ and $72 \mathrm{~h}$ and then filtered to separate BGs conditioned-
Commented [A1]: The in vivo study need repeat in the future, should I delete "systematically?)

Commented [A2R1]: Yes.

Formatted: Font: Italic

Commented [A3]: Don't forget the reference.

A unified in vitro evaluation for apatite-forming ability of bioactive glasses and their variants, Journal of Materials Sciences: Materials in Medcine 2015, DOI 10.1007/s10856015-5403-9

Formatted: Font: $12 \mathrm{pt}$ 
culture media and solids. The $\mathrm{pH}$ of the extracted BGs culture media was measured using a pH meter (OHAUS, China)START ER5000). Two separate samples from each immersion point of interest of all studied glasses-were prepared for all studied glasses.

The apatite formation ability of studied bioactive glasses in $\alpha$-MEM cell culture medium was evaluated using FTIR ALPHA II (Bruker, Germany). The extracted cell culture media containing ions released from BGs were diluted by a factor of 10 in deionized water for the ion concentration quantification of calcium, silicon and phosphorus using an inductively coupled plasma optical emission spectrometer (ICPOES, PerkinElmer, USA). The ions concentration of each BGs conditioned-culture medium was measured in triplicate,for three times, the Mean \pm SD of the total-6 measurements were presented as measured ion concentration for the BG conditionedculture medium glass-at the immersion point of interest.-

2.4 The investigation of osteogenesis of osteoblasts in vitro

The filtered $-4 \mathrm{~h}$ BG conditioned $\alpha$-MEM media were chesen and-sterilized by passing through a $0.2 \mu \mathrm{m}$ filter and supplemented with $10 \%(\mathrm{v} / \mathrm{v})$ fetal bovine serum (FBS) and $1 \%(\mathrm{v} / \mathrm{v})$ penicillin/streptomycin [153]-. The were preparedd as BGs conditioned culture media were used for the following in vitro investigation of osteogenic and angiogenic effects.

\subsubsection{Cell culture}

Pre-osteoblasts (MC3T3-E1) cells purchased from Shanghai cell bank (ATCC) were incubated in $\alpha$-MEM supplemented with $10 \%$ (v/v) fetal bovine serum (FBS) and $1 \%$ $(\mathrm{v} / \mathrm{v})$ penicillin/streptomycin [1135] in a humidified $\mathrm{CO}_{2}$ incubator at $37^{\circ} \mathrm{C}$. Culture medium was changed and replenished with fresh medium every two days till cells grew to $80 \%-90 \%$ confluence.

\subsubsection{Metabolic Cell viabilityactivity of MC3T3-E1cells}

Cell metabolic activity was estimated using Cell Counting Kit-8 assay. The MC3T3E1 cells were seeded into 96-well plates at a density of $1 \times 10^{3}$ cells/well and incubated at $37^{\circ} \mathrm{C}$ overnight. Cells were treated with extract BGs conditioned culture media and the control group ( $\alpha$-MEM with supplements). After 1, 3 and $7 \mathrm{~d}$ incubation, cell viability was assessed by the cell counting kit-8 (CCK-8) test according to the manufacturers' instructions (Meilunbio ${ }^{\circledR}$, DalianChina), and the absorbance was quantified at $450 \mathrm{~nm}$ using a UV-Vis Absorption Spectroscopy (Bio Terk, USA).

2.4.3 Quantitative alkaline phosphatase activity (ALP)

ALP activity was determined by enzyme histochemical assay [1146] . After 7, 10 and $14 \mathrm{~d}$ culture, the cells were lysed by freezing and thawing, and then reacted with 2.5 $\mathrm{mg} / \mathrm{mL}$ of 4-nitrophenyl phosphate disodium hexahydrate containing $1 \mathrm{mM} \mathrm{MgCl} 2$ (pH $=9.5$ ) for $1 \mathrm{~h}$. The reaction was finally stopped with $0.5 \mathrm{M} \mathrm{NaOH}$ and the absorbance was quantified at $405 \mathrm{~nm}$.

2.4.4 Mineralization
Commented [A4]: Shall we insert company info and country?

Formatted: Normal, Justified, Indent: Left: 0", First line: 0", Space Before: $0 \mathrm{pt}$, After: $0 \mathrm{pt}$, Pattern: Clear, Tab stops: Not at $0.64^{\prime \prime}+1.27^{\prime \prime}+1.91^{\prime \prime}+2.54 "+3.18^{\prime \prime}+3.82^{\prime \prime}+$ $4.45^{\prime \prime}+5.09 "+5.73^{\prime \prime}+6.36^{\prime \prime}+7 "+7.63^{\prime \prime}+8.27^{\prime \prime}+8.91^{\prime \prime}$ $+9.54 "+10.18 "$ 
The mineralization abilities of BGs with different phosphorus contents (0-5.07 mol\%) were assessed by Alizarin Red S staining. Cells were cultured in extracted BG conditioned culture media with $50 \mu \mathrm{mg} / \mathrm{ml} \mathrm{L}$-ascorbic acid and $5 \mathrm{mM} \beta$

b-glycerophosphate for 14 to $28 \mathrm{~d}$. Plates were then stained with $2 \%$ Alizarin Red S (Solarbio, BeijingChina) accordingly [1215] and the absorbance of the resulting solution was measured at $570 \mathrm{~nm}$.

\subsubsection{Quantitative real-time polymerase chain reaction (RT-qPCR)}

After 4, 7, 14 and $21 \mathrm{~d}$ culture, the expression of osteogenic genes (OPN, OCN and RUNX2) and angiogenic genes (VEGF) were evaluated by RT-qPCR as described by Liu and team- [12]. Briefly, according to the manufacturer's instruction, ReverTra Ace qPCR RT Master Mix reagents (TOYOBO, Japan) were used to reverse RNA into cDNA. Subsequently, $100 \mathrm{ng}$ of total cDNA was applied for RT-qPCR analysis using KOD SYBR ${ }^{\circledR}$ qPCR Mix reagents (TOYOBO, Japan). Finally, the relative expression was calculated using $\Delta \Delta \mathrm{Ct}$ method against endogenous reference, GAPDH. The fold change was determined from $2^{-\Delta \Delta \mathrm{Ct}}$. All primer sequences were presented in Table S1. Note that the genes expression of control group at different culturing dateys wereas set as 1 respectively. and $t$ The expression level of the BGs-contained culture media were normalized toand compared against the control atwith the same culturing days.

\subsubsection{Western blotting}

MC3T3-E1 cells were treated with extracted BGs conditioned culture media for 7, 14 and $21 \mathrm{~d}$. At the end of the treatment, cells were collected and lysed with RIPA lysate buffer (Beyotime, China) containing protease inhibitor. The BCA protein assay kit (Thermo Scientific, USA) was used to analyze the protein concentrations. Same amount of protein by weight was subjected to Sodium Dodecyl Sulphate-Poly Acrylamide Gel Electrophoresis (SDS-PAGE) and then transferred onto polyvinylidene difluoride (PVDF) membrane (Millipore, USA). The membranes were blocked in 3\% BSA and incubated with primary antibody (Abcam, UK) at $4{ }^{\circ} \mathrm{C}$ overnight and then HRPconjugated secondary antibody (Abcam, UK) at room temperature for $1 \mathrm{~h}$. Protein bands were detected with a Super Sensitive ECL Luminescence Reagent (Mmeilunbio, Dalian China) and visualized on an enhanced chemiluminescence (ECL) detection system (Tanon, China). The optical densities of the bands were measured by ImageJ software. GAPDH was used as a loading control. The protein secretion level of control group at different culturing datey wereas set as 1 respectively, while the protein concentration of the BGs were normalized and compare against to-the control atwith the same culturing days.

\subsubsection{Immunofluorescence staining}

After 7 and $14 \mathrm{~d}$ in culture, cells were fixed with $4 \%$ paraformaldehyde (PFA) for 15 min, blocked with $3 \%$ BSA in PBST at $4^{\circ} \mathrm{C}$ overnight and then incubated with OPN antibody for $1 \mathrm{~h}$ at room temperature. Cells were washed with PBST for 3 times and incubated with Alexa Fluor ${ }^{\circledR} 455$ (green) goat anti-rabbit $\operatorname{IgG}(\mathrm{H}+\mathrm{L})$ antibody for $1 \mathrm{~h}$ at room temperature. After further washing with PBST for 3 times, cells were labelled
Formatted: Default Paragraph Font, Font: (Default) SimSun Formatted: Justified, Space Before: $0 \mathrm{pt}$, After: $0 \mathrm{pt}$, No widow/orphan control 
DENTMA-D-20-00341, R1

with 4, 6-diamino-2-phenyl indole (DAPI) in PBS for 5 min. Finally, the fluorescent images were photographed by a fluorescent microscope (Carl Zeiss, Thuringia, Germany). 


\subsubsection{Surgical procedures.}

The animal experiment was approved by the Animal Research Ethics Committee of the Third Xiang Ya Hospital of Central South University (Certificate No 2019SYDW0154). Six male Sprague-Dawley (SD) rats (220-250 g in weight, 8-10 w old) were randomly divided into 2 groups (P0.00 group and P5.07 group) for an eight-week in vivo study. We followed the surgical procedures as described previously [1167, 117 $\underline{1}$ ]. Briefly, Rats were anesthetized with intraperitoneal injection of $1 \%$ sodium pentobarbital (3.5 $\mathrm{mg} / 100 \mathrm{~g}$ ), around $1.5 \mathrm{~cm}$ sagittal incision was made in the scalp and the calvarium was exposed by blunt dissection. Two critical size calvarial defects ( $5 \mathrm{~mm}$ in diameter) with distance of 4-5 $\mathrm{mm}$ in the central area of each parietal bone were created using a dental bone drill. BG granules were implanted in the created defects accordingly with and the soft tissues were sutured. After surgery, 80,000 units of penicillin were injected intramuscularly to prevent infection. The rats were sacrificed at $8 \mathrm{w}$ post-surgery and the parietal bones with BG granules were harvested and fixed in $4 \%$ paraformaldehyde solution for further micro-CT scan.

\subsubsection{Micro-computed tomography (micro-CT)}

The evaluation of new bone formation in rat calvarial defects after $8 \mathrm{w}$ post-surgery was conducted using a micro-CT (SCANCO MEDICAL AG/vivaCT80, Switzerland). Brifely, the settings of X-ray tube were $55 \mathrm{kV}$ and $145 \mu \mathrm{A}$. The harvested bone specimens were scanned at a resolution of $11.4 \mu \mathrm{m}$ and - a $5.0 \mathrm{~mm}$ in diameter region of the interest (ROI) was defined to evaluate the extent of bone formation. SubsequentlyAfter 3D reconstruction, the new bone volume to total bone volume (BV/TV) were analyzed using the SkyScan software, accordingly [19]. how to define the new bone, how to calculate the bone volume and were BG granules visualized in microCT scanning [ref]

\subsection{Statistical analysis}

Data were presented as mMean \pm standard deviation (SD). One-way analysis of variance (ANOVA) was used to perform statistical analysis. A value of $p<0.05$ was considered as statistically significant.

\section{Results}

\subsection{Material characterization and apatite formation}

XRD patterns (Fig. 1a) show the characteristic amorphous halo with no signs of crystallization peaks for all studied BGs indicating the glassy nature of the materials. FTIR also demonstrate typical glassy spectra (Fig. S1). This is in agreement with the previous studies of O'Donnell et al. [9]. Glass transition temperature $\left(\mathrm{T}_{\mathrm{g}}\right)$ extracted from DSC traces were plotted against phosphate content in the BGs as shown in Fig. 1b. It is clear that $T_{g}$ decreases with an increase in phosphate content which are also in good agreement with those measured by O'Donnell et al. [2018], suggesting that the 
synthesized glasses are the same as what O'Donnell achieved so that the results obtained on this work can be compared with the in vitro study carried out previously [2018]. The slight difference in values could be attributed to the use of different DSC instruments.

FTIR spectra of the studied BGs after $72 \mathrm{~h}$ immersion in $\alpha$-MEM (Fig. 1c) are obviously different to those before immersion (Fig. S1). Compared to the initial glasses, the intensity of the non-bridging oxygen bond at $920 \mathrm{~cm}^{-1}$ decreased significantly, indicating the studied glasses degraded. Additionally, the appearance and the sharpening of the typical apatite split peaks at the wavelengths of 600 and $560 \mathrm{~cm}^{-1}$ and the crystallized orthophosphate band at $1035 \mathrm{~cm}^{-1}$, suggest rapid apatite formation. It was noted that the increase of the phosphate content in the BGs led to more pronounced split peaks associated with faster and more apatite formation, similar to what has been previously observed in SBF [9].

\subsection{Ion release and $\mathrm{pH}$ change}

The concentration of $\mathrm{Ca}, \mathrm{P}$ and $\mathrm{Si}$ in $\alpha$-MEM culture media after immersion are shown in Fig.2a to 2c. The measured calcium concentration in all BG conditioned-culture media increased rapidly in the first $4 \mathrm{~h}$, indicating rapid glass degradation. The glasses with low phosphate content $(\leq 1.02 \mathrm{~mol} \%)$ demonstrated a slight fluctuation of $\mathrm{Ca}$ concentration between 4 and $24 \mathrm{~h}$ followed by a clear reduction, while the high phosphate containing BGs (P3.62 and P5.07, 3.62 and 5.07 mol\% $\mathrm{P}_{2} \mathrm{O}_{5}$ ) showed an increase in the first $4 \mathrm{~h}$ followed by a gradual decrease in Ca concentration. These two distinctly different behaviors might be due to the fact that the low $\mathrm{P}$ limited the formation of the apatite layer and therefore the amount of $\mathrm{Ca}$ consumed was lower(lower than the amount released from BGs), consequentially an increase in the $\mathrm{Ca}$ concentration (Fig. 2a) in the low phosphate containing BGs. In the case of measured $\mathrm{P}$ concentration, in contrast to the continuous reduction observed in low phosphate containing BGs, the high phosphate containing BGs (P3.62 and P5.07) showed a slight increase in $\mathrm{P}$ concentration from 0 to $4 \mathrm{~h}$ followed by a decrease with prolonged immersion (Fig. 2b). The decrease in $\mathrm{P}$ concentration observed in both cases was a result of $\mathrm{P}$ depletion as a result of apatite formation [2119]. A clear increase in Si concentration was found for all the glass compositions up to $24 \mathrm{~h}$ of immersion. A small reduction was found between 24 to $72 \mathrm{~h}$. Although not significantly different, glasses with a higher $\mathrm{P}$ content showed a higher Si concentration at all investigated time points (Fig. 2c). This data suggested that the studied BGs degraded rapidly in $\alpha$-MEM and high $\mathrm{P}$ content might facilitate glass degradation.

Fig. 2d shows the variation in $\mathrm{pH}$ of BGs-conditioned media plotted against immersion time. As a general trend, $\mathrm{pH}$ increased significantly in the first $12 \mathrm{~h}$, decreased at $24 \mathrm{~h}$ and increased slightly afterwards. It was clear that the $\mathrm{pH}$ rise was less pronounced for the high phosphate content glasses compared to the low phosphate content glasses as a result of the acidic phosphate buffering the alkaline $\mathrm{pH}$ generated by the silica glass network degradation. A similar trend was seen in SBF by O'Donnell [9].
Formatted: Font: Italic 


\subsection{Cell viability and ALP activity}

After 1, 3 and $7 \mathrm{~d}$ in culture, cells treated with culture media containing ions released from the BGs after $4 \mathrm{~h}$ immersion clearly showed a greater cell viability than that treated with control culture medium $(\mathrm{p}<0.05)$ (Fig. 3a). The cell viability was enhanced with longer culture times for all glass compositions, while there was no obvious difference among the experimental groups.

After 7, 10 and $14 \mathrm{~d}$ in culture, the ALP activities of MC3T3-E1 cultured with extracted BGs media were significantly higher than that with control medium (Fig. 3b). This indicated that the culture media containing ions released from studied BGs has a function of promoting cell differentiation. Compared to glass without the presence of phosphate (P0.00), glass P5.07 has a lower ALP activity at 10 and $14 \mathrm{~d}(\mathrm{p}<0.05))=$. It is possible that the high phosphate content BG (5.07 mol\%) accelerated the cell growth cycle as shown at 7d that a higher cell viability was seen for P5.07 compared to

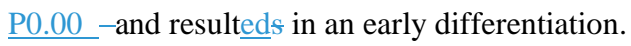

\subsection{Mineralization ability}

The mineralization potential of MC3T3-E1 cells in BGs-conditioned media was evaluated using Alizarin Red $\mathrm{S}$ staining. As can be seen from the qualitative and quantitative analysis of staining in Fig. 4, the MC3T3-E1 cells treated with BGs conditioned media showed a stronger staining compared to the cells cultured with control medium $(\mathrm{p}<0.05)$ after 14,21 and $28 \mathrm{~d}$. Moreover, a marked effect on enhancing staining was seen in the phosphate containing BGs in comparison to that in the phosphate free BG, confirming that phosphate facilitates mineralization and bone nodule formation in vitro.

\subsection{Gene expression}

The effect of BGs conditioned-culture media on the regulation of osteogenic genes expression of MC3T3-E1 cells was conducted by RT-qPCR. After over $21 \mathrm{~d}$ in culture, the expression of OPN gene increased significantly in the BGs conditioned culture media, especially in the phosphate containing BGs compared with that in control medium (Fig. 5a) indicating that phosphate released from BGs stimulated osteoblastic differentiation. This is well in line with the obtained Alizarin Red S staining data presented in Fig. 4. In the case of OCN (Fig.5b), either lower expression or no significant difference was found in the BGs conditioned culture media against the control group. This might be attributed to the insufficient culture time for OCN expression. Tsigkou et al. [2119] found that OCN is a marker of the late stages of osteoblast differentiation and its production denotes the onset of matrix deposition. Fig.5c showed that a higher expression of RUNX2 in BGs conditioned culture media than that in the control media was only seen at $7 \mathrm{~d}$, while the opposite was observed at 4, 14 and 21 d. A less significant down-regulated effects on both OCN and RUNX2 were observed in the glasses with phosphate at $21 \mathrm{~d}$ in culture. Phosphate appeared to stimulate osteogenic genes up-regulation or inhibite osteogenic genes down-regulation. 
VEGF is known as a key angiogenic factor, which possesses significant biological activity in enhancing blood vessel formation [20]22] which is critical for the repair of larger $(>2 \mathrm{~mm}$ ) bone defects. Compared to that in the control medium, the expression of VEGF was lower in all BGs treated groups at $4 \mathrm{~d}$, however the down-regulated effect reduced with an increase in phosphate content (Fig. 5d) in BGs group. With an increase in culturing time up to $21 \mathrm{~d}$, the BGs conditioned media initiated a stimulative effect on VEGF expression. After $21 \mathrm{~d}$, cells treated with glasses containing 3.62 and 5.07 mol\% $\mathrm{P}_{2} \mathrm{O}_{5}$ demostrated significantly higher $(\mathrm{p}<0.05)$ VEGF gene expression than that in phosphate free bioactive glass, indicating that phosphorus may play an important role in vascularization.

\subsection{Western blot analysis}

To further investigate the effect of phosphate containing BGs on osteogenic and angiogenic differentiation of osteoblasts, the modulation of osteogenic and angiogenic related proteins OPN, RUNX2 and VEGF were determined by western blot. As can be seen in Fig. 6a, after 7, 14 and $21 \mathrm{~d}$ culturing, compared to the control medium, the OPN and VEGF protein levels were generally enhanced in BGs conditioned culture media, while an increase in RUNX2 level was noticed at $7 \mathrm{~d}$, but no significant difference for long period treatment (14 and $21 \mathrm{~d}$ ). These results are consistent with the gene expression data shown in Fig.5 suggesting that phosphate in BGs plays a key role in promoting osteoblast differentiation and vascularization.

\subsection{Immunofluorescence (IF) staining}

OPN is a major protein related to the production of extracellular matrix [2321], which could further promote mineralization [21[19]. To further study the effect of phosphate in BGs on osteogenic differentiation of the MC3T3-E1 cells in vitro, the secretion of osteogenic protein OPN at 7 and $14 \mathrm{~d}$ were semi-quantified by immunofluorescence staining. In the early stage ( $7 \mathrm{~d})$, in comparison to the control group that barely showed OPN protein secretion, the cells treated with BGs conditioned culture media exhibited more visible OPN expression (Fig.7). Meanwhile, the glass with $5.07 \mathrm{~mol} \% \mathrm{P}_{2} \mathrm{O}_{5}$ showed higher OPN secretion than $45 \mathrm{~S} 5\left(2.6 \mathrm{~mol} \% \mathrm{P}_{2} \mathrm{O}_{5}\right)$ that showed higher OPN secretion than the glass without the presence of phosphate. Moreover, it is worth noting that at $7 \mathrm{~d}$ culture, OPN protein was located in both extracellular matrix (ECM) and nucleus, implying that the cells were secreting OPN protein, while at $14 \mathrm{~d}$ OPN was mainly localized in the ECM.

\subsection{Investigation of osteogenesis in vivo}

The new bone formation in rat calvarial defects after $8 \mathrm{w}$ treatment with BGs granules were visualized by the reconstructed Micro-CT images as shown in Fig. 8. A larger amount of new bone formation was visible from the defect implanted with BG containing 5.07 mol\% $\mathrm{P}_{2} \mathrm{O}_{5}$ compared with that implanted with the $\mathrm{BG}$ with no $\mathrm{P}_{2} \mathrm{O}_{5}$. 
The BV/TV for the defect implanted with BG P5.07 is higher than that of BG P0.00 $(19.90 \pm 10.05 \%$ vs $17.06 \pm 12.08 \%)$. Whilst not significantly different theses results are in agreement with the findings obtained from cell studies that $\mathrm{P}$ content played a positive role on osteogenesis and suggested that phosphate favored new bone formation and facilitated osseointegration.

\section{Discussion}

The role of phosphorus in silicate glass structure, bioactivity and bone mineralization potential is controversial. Initially, it was believed that phosphorus enters the silicate glass network by forming Si-O-P bonds [2224]. More recently, O'Donnell and Hill [10] designed silicate glasses with varying phosphate content $(0-9.25 \mathrm{~mol} \%)$ and sufficient cations that charge balance the phosphate to study the role of phosphorus using both ${ }^{31} \mathrm{P}$ and ${ }^{29} \mathrm{Si}$ MAS-NMR. It was found that phosphorus does not enter the silicate network but exists as a separate orthophosphate species, which showed a good agreement with findings in literature [25-2723 25]. O'Donnell et al. [9] also found that an enhanced bioactivity can be achieved in SBF by increasing phosphate content, furthermore, the phosphate content has more significant impact than NC on bioactivity.

In this study, FTIR and ICP-OES results (Fig. 1c and Fig. 2) confirmed that the high phosphate content led to a superior bioactivity in culture media in a similar fashion to that in SBF [9]. The change of Ca, P, Si concentration in the BG conditioned-culture media was a joint results of glass dissolution and apatite formation. A significant increase in $\mathrm{Ca}$ and $\mathrm{Si}$ ion concentrations were observed at $4 \mathrm{~h}$ after immersion unequivocally revealed rapid glass degradation of all the studied glasses. This is supported by the dramatic increase in $\mathrm{pH}$, which was a result of ion exchange between $\mathrm{Na}^{+}$or $\mathrm{Ca}^{2+}$ from BGs and $\mathrm{H}^{+}$ions from culture media. Despite that $\mathrm{P}$ free $\mathrm{BG}$ has a higher Si content, a higher Si concentration was seen from the glasses with a higher phosphate content which might imply the potential of promoting glass degradation by increasing the phosphate content. Alternatively Alternatively, the increase in the Si concentration could simply reflect the greater solubility of silicates at pHs closer to $\mathrm{pH} 7$. The rapid silica-gel formation could provide sites for the subsequent apatite formation [28[26]. Additionally, the released Si is also believed to play an essential role in bone formation by being responsible for the cell-stimulating activities [2729]. With BGs degradation, the measured phosphate in culture media was expected to behave in a similar faction to that of $\mathrm{Ca}$ and $\mathrm{Si}$, however, the concentration of $\mathrm{P}$ in culture media decreased for glasses with low phosphate content $(\leq 1.02 \mathrm{~mol} \%)$ with an increase in immersion time, in contrast, measured $\mathrm{P}$ concentration increased to a maximum at $4 \mathrm{~h}$ and then decreased for high phosphate content BGs $(\geq 3.62 \mathrm{~mol} \%))$. This is attributed to fast apatite formation upon immersion, which consumed the $\mathrm{P}$ from the culture media or that was released from BGs, in another word the amount of P released from low phosphate content glasses is less than that was consumed for apatite formation.

Fast apatite formation is also evidenced by the reduction of $\mathrm{Ca}$ concentration and $\mathrm{pH}$ changes in Fig 2a and 2d, respectively. The studied BGs (Table 1) have a ratio of $\mathrm{Ca} / \mathrm{P}$ higher than that in hydroxyapatite, which is close to 1.67 , therefore the apatite 
formation upon immersion is determined by the phosphate content in the glass compositions. FTIR spectra in Fig. 1b showed a faster apatite formation for the glass with higher phosphate content. This data suggests that high phosphate content leads to a superior bioactivity in vitro. A similar effect was also shown by Mneimne et al. [28][30] that an increase in phosphate content in the fluoride containing bioactive glass favors apatite formation rather than fluorite $\left(\mathrm{CaF}_{2}\right)$ formation-. The formed apatite layer provides an ideal environment for osteoblast adhesion, proliferation and differentiation [3129]. Moreover, faster apatite layer formation could increase the biological activity in vivo [3230].

Based on the $\mathrm{pH}$ and ICP-OES results, the $4 \mathrm{~h}$ BG conditioned $\alpha$-MEM showed the highest $\mathrm{pH}$ level and ion concentrations represents the highest cytotoxicity risk to cell growth, therefore, the filtered $4 \mathrm{~h} \mathrm{BG} \alpha$-MEM media was selected for the-cell study. The cell viability of osteoblasts, MC3T3-E1 cells treated with culture media containing ions released from BGs was significantly greater than that treated with control culture medium alone suggesting that the ions released via dissolution of all the studied BGs were non-cytotoxic, confirming the promising potential these BGs for bone repair and promoting osteoblast proliferation. This is in agreement with previous studies that showed free ions such as $\mathrm{Ca}^{2+}, \mathrm{PO}_{4}{ }^{3-}$ released from $\mathrm{BGs}$ accelerated the proliferation of cells [3129]. It is worth noting that DNA quantification could provide evidence of cell proliferation and complementary results for CCK-8 test. Wu and team [3331] found that the dissolution of $\mathrm{BG}$ which released $\mathrm{Si}, \mathrm{Ca}, \mathrm{Na}$ and $\mathrm{P}$ ions could accelerate the osteoblasts cell cycle through the transition from G0 to G1 stages, and also promote cell proliferation in S and G2-M phases.

Our data showed that the extracted BGs treated culture media not only promoted MC3T3-E1 cell proliferation, but also stimulated cell differentiation (Fig 3b). Compared to the treatment with control culture medium, the ALP activity of MC3T3E1 cells treated with extracted BGs culture media was much higher at all studied time points. The increase in ALP activity is typically seen at an early stage of osteogenic differentiation to promote extracellular matrix maturation and ALP was also found to split $\mathrm{P}-\mathrm{O}-\mathrm{P}$ to release $\mathrm{PO}_{4}{ }^{3-}$ and drive apatite formation, and the increase was therefore considered as an important indicator for early osteogenesis [34[32]. After 10 and $14 \mathrm{~d}$ in culture, the high phosphate containing glass P5.07 showed a lower ALP activity compared to glass P0.00 (Fig. 3b). This is highly likely due to the fact that high phosphate concentration accelerated the osteoblast cell cycle and resulted in an earlier entrance to the calcification stage. It is supported by the observed bone nodules formation evaluated by alizarin red staining in Fig. 4, which showed that the BGs with higher phosphate content demonstrated a more significant effect on enhancing staining, suggesting that phosphate facilitates bone mineralization.

It is known that RUNX2, OPN, OCN and VEGF are crucial genes associated with critical roles in mineralization [3533]. The RT-qPCR and western blot analysis demonstrated an increase in the expression of OPN and VEGF upon treatment over time in BGs conditioned culture media in comparison to control media, while a reverse
Formatted: Font: Italic

Formatted: Font: Italic 
trend was generally seen for RUNX2 and OCN at $21 \mathrm{~d}$. It was noted that, compared to phosphate free BG, the phosphate containing BGs conditioned media showed a facilitating effect on up-regulating OPN and VEGF expression and an inhibiting effect on down-regulating RUNX2 and OCN genes expression at $21 \mathrm{~d}$ in culture. Immunocytochemistry analysis confirmed the expression of OPN protein increased with an increase in phosphate content in BGs. Moreover, the localization of OPN was found in both cytosol and nucleus at $7 \mathrm{~d}$. The 45S5 Bioglass ${ }^{\circledR}$ has been used in orthopedics and dentistry since 1985 attributed to its good osteogenic competence [8]. However, the glass with $5.07 \mathrm{~mol} \% \mathrm{P}_{2} \mathrm{O}_{5}$ showed a comparable or more obvious inducible effect on the expression of osteogenic markers (Fig. 6 and Fig. 7), which further improve mineralization. Taken together, the interaction between osteoblasts and BGs conditioned culture media with ions released from studied glasses suggested that the studied soda-lime-phospho-silicate glasses are able to induce the differentiation of osteoblast and promote the mineralization potential of osteoblast in a phosphate dependent manner.

In order to get a closer insight on the effect of phosphorus in BGs on osteogenesis in a dynamic system to mimic that in human body and better estimate their clinical success, a pilot in vivo study on the ability of high phosphate content BG to repair a criticalsized calvarial defect in rat was conducted. It was likely that all the bioactive glasses have degraded after $8 \mathrm{w}$. Moreover, tThe obtained results revealed that high phosphate content BG (P5.07) enhanced bone formation in rat calvarial defect at $8 \mathrm{w}$ compared to the BG with the absence of phosphate. This is highly likely attributed to the crucial physic-chemical and biological functions of phosphorus, including reducing the $\mathrm{pH}$ rise associated with ion exchange during glass degradation, speed up ion release, facilitate apatite formation [9,-3028], increase cell viability and ALP activity, and promote osteogenic and angiogenic gene and protein expression etc. A matched glass degradation with new bone formation is crucial clinically. An increase in glass granule $\underline{\text { size could compromise undesired rapid glass degradation. Micro-CT results obtained }}$ in the present study provide evidence for in vivo glass degradation and new bone formation. Histological evalution would provide complemetry information and will be carried out In vive investigation of the effect of phosphate in BGs on osteogenesis including Micro-CT and histological evaluations will be conducted-systematically on both small and large animal models alongside micro-CT analysis in future work.

\section{Conclusions}

To the best of our knowledge, this is the first study on the effect of varying phosphate content in soda-lime-phospho-silicate bioactive glasses on osteogenesis. The obtained results showed that glasses with a higher phosphate content formed apatite faster in $\alpha$ MEM culture media in a similar manner to that in SBF. The studied glasses not only possess excellent bioactivity but also promising osteogenesis. With an increase in phosphate content in BGs, the BGs conditioned culture media led to an increase in the ALP activity of MC3T3-E1 cells, induced the expression of OPN, VEGF, while weakened the down-regulation of RUNX2 and OCN genes expression. 
The in vivo study demonstrated that high phosphate content BG (P5.07) enhanced bone formation in rat calvarial defect at $8 \mathrm{w}$ comparing to phosphate free BG (P0.00). Therefore, there is a positive correlation between the phosphate content in BGs and glass bioactivity and osteogenesis efficacy. These findings connected the bioactivity study in SBF and culture media, cell study and animal study together and provided a better understand of the relationship between phosphate content in BGs and the biological effects that is currently lacking in the published literature.

The results of the present study demonstrate that that high phosphate content in BGs

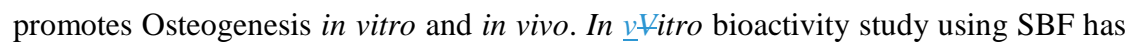
similar results as that used culture media indicating conventional bioactivity study using SBF can provide valuable indication in designing new bioactive glasses for bone repair or replacement in Medicine and Dentistry.

\section{Acknowledgments}

This study was supported by National Natural Science Foundation of China (81701015), Natural Science Foundation of Hunan Province, China (2019JJ30018), Changsha Prominent Young Innovators Program (kq1905059), and Huxiang Youth Talent Program of Hunan Province of China (2020RC3064) the Open Sharing Fund for the Largescale Instruments and Equipment of Central South University (CSUZC201939) and Harbin Science and Technology Bureau Project (2016AE4AE004).

The authors would like to acknowledge the use of the Department of Materials X-ray Diffraction Suite at the University of Manchester and for the technical support, advice and assistance provided by Dr. John E. Warren. 


\section{Reference}

[1] V. Wu, M.N. Helder, N. Bravenboer, C.M. ten Bruggenkate, J. Jin, J. Klein-Nulend, E.A.J.M. Schulten, Bone Tissue Regeneration in the Oral and Maxillofacial Region: A Review on the Application of Stem Cells and New Strategies to Improve Vascularization, Stem Cells International 2019 (2019) 1-15.

[2] G. Fernandez de Grado, L. Keller, Y. Idoux-Gillet, Q. Wagner, A.M. Musset, N. Benkirane-Jessel, F. Bornert, D. Offner, Bone substitutes: a review of their characteristics, clinical use, and perspectives for large bone defects management, Journal of Tissue Engineering 9 (2018) 1-18.

[3] A.R. Amini, C.T. Laurencin, S.P. Nukavarapu, Bone Tissue Engineering: Recent Advances and Challenges, Critical Review in Biomedical Engineering 40 (5) (2012) 363-408.

[4] J. Wu, G. Miao, Z. Zheng, Z. Li, W. Ren, C. Wu, Y. Li, Z. Huang, L. Yang, L. Guo, 3D printing mesoporous bioactive glass/sodium alginate/gelatin sustained release scaffolds for bone repair, Journal of Biomaterials Applications 33 (6) (2019) 755-765. [5] L.L. Hench, R.J. Splinter, W.C. Allen, T.K. Greenlee, Bonding mechanisms at the interface of ceramic prosthetic materials, Journal of Biomedical Materials Research 2 (1) (1971) 117-141.

[6] L.L. Hench, The story of Bioglass, Journal of Materials Science: Materials in Medicine 17 (11) (2006) 967-978.

[7] L.L. Hench, I.D. Xynos, J.M. Polak, Bioactive glasses for in situ tissue regeneration, Journal of Biomaterials Science Polymer Edition 15(4) 543-562.

[8] L.L. Hench, J.R. Jones, Bioactive Glasses: Frontiers and Challenges, Frontiers in Bioengineering Biotechnology 3 (2015) 194.

[9] M.D. O'Donnell, S.J. Watts, R.G. Hill, R.V. Law, The effect of phosphate content on the bioactivity of soda-lime-phosphosilicate glasses, Journal of Materials Science: Materials in Medicine 20 (8) (2009) 1611-1618.

[10] M.D. O'Donnell, S.J. Watts, R.V. Law, R.G. Hill, Effect of $\mathrm{P}_{2} \mathrm{O}_{5}$ content in two series of soda lime phosphosilicate glasses on structure and properties - Part I: NMR, Journal of Non-Crystalline Solids 354 (30) (2008) 3554-3560.

[11] M. Edén, The split network analysis for exploring composition-structure correlations in multi-component glasses: I. Rationalizing bioactivity-composition trends of bioglasses, Journal of Non-Crystalline Solids 357 (2011) 1595-1602.

[12] J. Liu, S.C.F. Rawlinson, R.G. Hill, F. Fortune, Fluoride incorporation in high phosphate containing bioactive glasses and in vitro osteogenic, angiogenic and antibacterial effects, Dental Materials Official Publication of the Academy of Dental Materials 32 (2016) e221-e237.

[13] A. L. B. Mac on , T.B. Kim, E.M. Valliant, K. Goetschius, R.K. Brow, D.E. Day, A. Hoppe, A.R. Boccaccini, Il-Y. Kim, C. Ohtsuki, T. Kokubo, A Osaka, M. Vallet-Reg', D. Arcos, L. Fraile, A.J. Salinas, A.V. Teixeira, Y. Vueva, R.M. Almeida, M. Miola, C Vitale-Brovarone, E Verne', W Ho land, J.R. Jones, A unified in vitro eval- uation for apatite forming ability of bioactive glasses and their variants, Journal of Materials Science: Materials in Medicine 2015, 26 (115). 
-[1432] X. Yan, X. Huang, C. Yu, H. Deng, Y. Wang, Z. Zhang, S. Qiao, G. Lu, D. Zhao, The in-vitro bioactivity of mesoporous bioactive glasses, Biomaterials 27 (18) (2006) 3396-3403.

[1543] W. Zhang, F. Zhao, D. Huang, X. Fu, X. Li, X. Chen, Strontium-Substituted Submicrometer Bioactive Glasses Modulate Macrophage Responses for Improved Bone Regeneration, ACS Applied Materials \& Interfaces 8 (45) (2016) 30747-30758. [1654] J.H. Lee, M.Y. Ryu, H.R. Baek, K.M. Lee, J.H. Seo, H.K. Lee, H.S. Ryu, Effects of porous beta-tricalcium phosphate-based ceramics used as an E. coli-derived rhBMP2 carrier for bone regeneration, Journal of Materials Science: Materials in Medicine 24 (9) (2013) 2117-2127.

_[15] J. Liu, S.C.F. Rawlinson, R.G. Hill, F. Fortune, Fluoride incorporation in high phosphate containing bioactive glasses and in vitro osteogenic, angiogenic and antibacterial effects, Dental Materials Official Publication of the Academy of Dental Materials 32 (2016) e221-e237.

[176] S. Zhao, J. Zhang, M. Zhu, Y. Zhang, Z. Liu, C. Tao, Y. Zhu, C. Zhang, Threedimensional printed strontium-containing mesoporous bioactive glass scaffolds for repairing rat critical-sized calvarial defects, Acta Biomaterialia 12 (2015) 270-280.

[187] H. Wang, S. Zhao, W. Xiao, X. Cui, W. Huang, M.N. Rahaman, C. Zhang, D. Wang, Three-dimensional zinc incorporated borosilicate bioactive glass scaffolds for rodent critical-sized calvarial defects repair and regeneration, Colloids and Surfaces B: Biointerfaces 130 (2015) 149-156.

[19],Y. Wang, X. Hu, L. Zhang, C. Zhu, J. Wang, Y. Li, Y. Wang, C. Wang, Y. Zhang, + Q. Yuan, Bioinspired extracellular vesicles embedded with black phosphorus for molecular recognition-guided biomineralization. Nature Communications 10, 2829 (2019).

[2018] M.D. O'Donnell, S.J. Watts, R.V. Law, R.G. Hill, Effect of $\mathrm{P}_{2} \mathrm{O}_{5}$ content in two series of soda lime phosphosilicate glasses on structure and properties - Part II: Physical properties, Journal of Non-Crystalline Solids 354 (30) (2008) 3561-3566.

[2119]_-O. Tsigkou, J.R. Jones, J.M. Polak, M.M. Stevens, Differentiation of fetal osteoblasts and formation of mineralized bone nodules by $45 \mathrm{~S} 5$ Bioglass ${ }^{\circledR}$ conditioned medium in the absence of osteogenic supplements, Biomaterials 30 (21) (2009) 35423550 .

[220] Z. Lin, J.S. Wang, L. Lin, J. Zhang, Y. Liu, M. Shuai, Q.I. Li, Effects of BMP2 and VEGF165 on the osteogenic differentiation of rat bone marrow-derived mesenchymal stem cells, Experimental and Therapeutic Medicine 7 (3) (2014) 625-629. [231] P. Naruphontjirakul, A.E. Porter, J.R. Jones, In vitro osteogenesis by intracellular uptake of strontium containing bioactive glass nanoparticles, Acta Biomaterialia 66 (2018) 67-80.

[242] G. Lusvardi, G. Malavasi, M. Cortada, L. Menabue, M.C. Menziani, A. Pedone, U. Segre, Elucidation of the Structural Role of Fluorine in Potentially Bioactive Glasses by Experimental and Computational Investigation, Journal of Physical Chemistry B 112 (40) 12730-12739.

[253] I. Elgayar, A.E. Aliev, A.R. Boccaccini, R.G. Hill, Structural analysis of bioactive
Formatted: Font: (Default) Times New Roman, Font color: Auto, Do not check spelling or grammar, Pattern: Clear Formatted: Normal

Formatted: Font: (Default) Times New Roman, Font color: Auto, Do not check spelling or grammar, Pattern: Clear

Formatted: Font: (Default) Times New Roman, Font color: Auto, Do not check spelling or grammar, Pattern: Clear

Formatted: Font: (Default) Times New Roman, Font color: Auto, Do not check spelling or grammar, Pattern: Clear

Formatted: Default Paragraph Font, Font: (Default) Times New Roman, Font color: Auto, Pattern: Clear

Formatted: Font: (Default) Times New Roman, Not Italic, Font color: Auto, Do not check spelling or grammar

Formatted: Default Paragraph Font, Font: (Default) Times New Roman, Font color: Auto, Pattern: Clear

Formatted: Font: (Default) SimSun 
glasses, Journal of Non-Crystalline Solids 351 (2005) 173-183.

[264] X. Chen, X. Chen, A. Pedone, D. Apperley, R.G. Hill, N. Karpukhina, New Insight into Mixing Fluoride and Chloride in Bioactive Silicate Glasses, Scientific Reports 8 (1) (2018)1-10.

[275] D.S. Brauer, N. Karpukhina, R.V. Law, R.G. Hill, Structure of fluoride-containing bioactive glasses, Journal of Materials Chemistry 19 (31) 5629-5636.

[286] D.S. Brauer, N. Karpukhina, M.D. O'Donnell, R.V. Law, R.G. Hill, Fluoridecontaining bioactive glasses: Effect of glass design and structure on degradation, $\mathrm{pH}$ and apatite formation in simulated body fluid, Acta Biomaterialia 6 (8) (2010) 32753282 .

[297] E.M. Carlisle, Silicon: An Essential Element For The Chick, Science 40 (7) (2009) 210-213.

[3028] M. Mneimne, R.G. Hill, A.J. Bushby, D.S. Brauer, High phosphate content significantly increases apatite formation of fluoride-containing bioactive glasses, Acta biomaterialia 7 (4) (2011) 1827-1834.

[3129] P.S.P. Poh, D.W. Hutmacher, B.M. Holzapfel, A.K. Solanki, M.M. Stevens, M.A. Woodruff, In vitro and in vivo bone formation potential of surface calcium phosphatecoated polycaprolactone and polycaprolactone/bioactive glass composite scaffolds, Acta biomaterialia 30 (2016) 319-333.

[320] N. Cozza, F. Monte, W. Bonani, P. Aswath, A. Motta, C. Migliaresi, Bioactivity and mineralization of natural hydroxyapatite from cuttlefish bone and Bioglass ${ }^{\circledR}$ cosintered bioceramics, Journal of tissue engineering and regenerative medicine 12 (2) (2018) 1131-1142.

[331] T. Wu, N. Cheng, C. Xu, W. Sun, C. Yu, B. Shi, The effect of mesoporous bioglass on osteogenesis and adipogenesis of osteoporotic BMSCs, Journal of Biomedical Materials Research A 104 (12) (2016) 3004-3014.

[3ㄹz] H. Hu, M. Chen, G. Dai, G. Du, X. Wang, J. He, Y. Zhao, D. Han, Y. Cao, Y. Zheng, D. Ding, An Inhibitory Role of Osthole in Rat MSCs Osteogenic Differentiation and Proliferation via Wnt/beta-Catenin and Erk1/2-MAPK Pathways, Cellular Physiology and Biochemistry 38 (6) (2016) 2375-2388.

[353] M. Huang, R.G. Hill, S.C.F. Rawlinson, Zinc bioglasses regulate mineralization in human dental pulp stem cells, Dental materials : official publication of the Academy of Dental Materials 33 (5) (2017) 543-552. 


\section{Captions to $\underline{\mathrm{t}}$ Tables and figures}

Table 1: Glass Compositions in mol\% designed by O'Donnell [9].

Formatted: Justified

Fig 1. (a) XRD patterns of initial glasses. (b) Glass Transition Temperature ( $\left.T_{g}\right)$ against $\mathrm{P}_{2} \mathrm{O}_{5}$ content in the BGs (匹: our data; $\bullet$ : data from O'Donnell [2018]). (c) FTIR spectra of BGs powders after $72 \mathrm{~h}$ immersion in $\alpha$-MEM.

Fig 2. Measured ion concentration in BGs conditioned $\alpha$-MEM at 4, 12, 24 and 72h (a) calcium, (b) phosphorous and (c) $\underline{s}$ Silicon; (d) $\mathrm{pH}$ variation of BGs-conditioned culture $\alpha$-MEM media. Note that where error bars are not seen, they are smaller than the data point.

Fig 3. (a) Cell viability, (b) ALP activity of BG-conditioned media on MC3T3-E1. Data are presented as the mean \pm standard error mean $(n=6)$. $* P<0.05$, compared with the control group. \#P $<0.05$, compared with glass $\mathrm{P} 0.00$.

Fig 4. (a) Representative images of MC3T3-E1 mineralization presented by Alizarin Red S staining after BG-conditioned culture media treatment. (b) Quantification of Alizarin Red S in MC3T3-E1 expressed as a fold change compared to the relative group. Data were presented as mean $\pm \mathrm{SD}(\mathrm{n}=3)$. $* \mathrm{P}<0.05$, compared with the control. \#P $<$ 0.05 , compared with glass P0.00.

Fig 5. The expression of (a) OPN, (b) OCN, (c) RUNX2 and (d)VEGF in MC3T3-E1 following BGs-conditioned culture media treatment up to $21 \mathrm{~d}$ analyzed by RT-qPCR. Data were presented as the mean $\pm \mathrm{SD}(\mathrm{n}=3)$. $* \mathrm{P}<0.05$, compared with the control. \#P $<0.05$, compared with $\mathrm{P} 0.001$ group. Note that the genes expression of control group at different culturing datey wereas set as 1 respectively and the expression level of the BGs-contained culture media were normalized and compared against to-the control group atwith the same culturing days.

Fig 6. (a) Representative images of VEGF, OPN and RUNX2 protein expression analyzed by western blot. (b) Relative intensities of VEGF, OPN and RUNX2 in reference to GAPDH were showed as a fold change in comparison to the control group. Data were presented as the mean $\pm \mathrm{SD}$ from three independent experiments. ${ }^{*} \mathrm{P}<0.05$, compared with the control. \#P $<0.05$, compared with $\mathrm{P} 0.004$ group. The protein secretion level of control group at different culturing datey wasere set as 1 respectively, while the protein concentration of the BGs groups was normalized and compared against the control atwith the same culturing days.

Fig 7. Representative fluorescence images show staining of DAPI (blue) and OPN (green) in MC3T3-E1 cells following BGs conditioned culture media treatment for (a) $7 \mathrm{~d}$ and (b) $14 \mathrm{~d}$. Scale bar $240 \mu \mathrm{m}$. The images in (a) and (b) were taken under low magnification and high magnification, respectively. 
DENTMA-D-20-00341, R1

Fig 8. Micro-CT evaluation of bone regeneration in the rat calvarial defects implanted with the BGs at $8 \mathrm{w}$ post-surgery. (a) Top and sagittal sectional views of reconstructed images. (b) Bone volume/total volume (BV/TV) in the defects implanted with P5.07 and $\mathrm{P} 0.00$. Data were expressed as mean $\pm \mathrm{SD}$ from three individual rats. 
DENTMA-D-20-00341, R1

Table 1: Glass Compositions in mol\% designed by O'Donnell [9].

\begin{tabular}{ccccc}
\hline Glass Code & $\mathbf{S i O}_{2}$ & $\mathbf{C a O}$ & $\mathrm{Na}_{2} \mathbf{O}$ & $\mathbf{P}_{2} \mathrm{O}_{5}$ \\
\hline P0.00 & 51.06 & 22.84 & 26.10 & 0.00 \\
P1.02 & 48.98 & 23.33 & 26.67 & 1.02 \\
P3.62 & 43.66 & 24.60 & 28.12 & 3.62 \\
P5.07 & 40.71 & 25.31 & 28.91 & 5.07 \\
\hline
\end{tabular}


DENTMA-D-20-00341, R1

\section{Figures for paper}

A

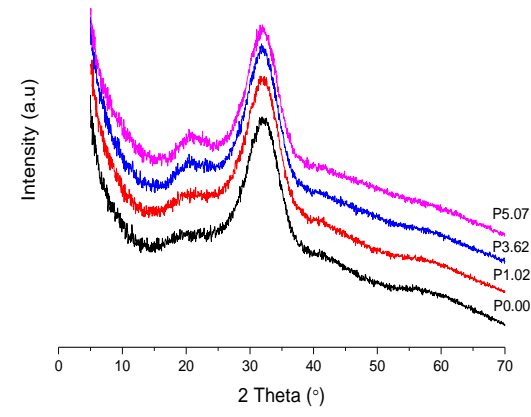

C
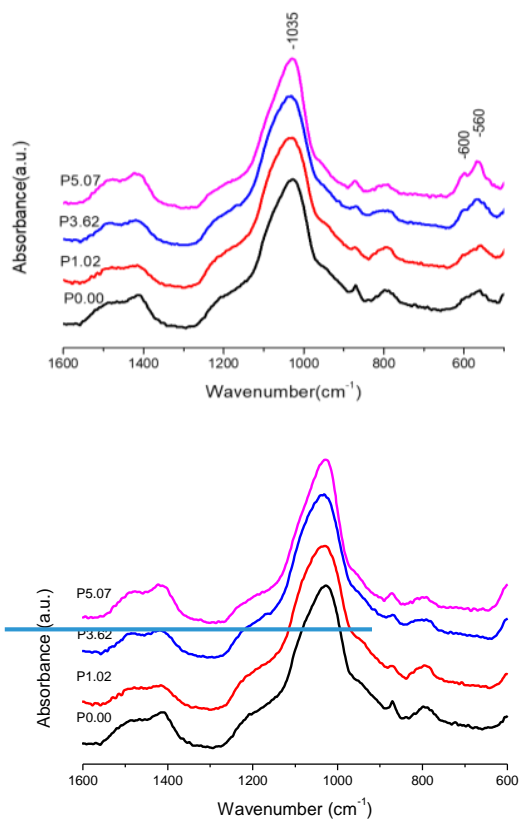

B

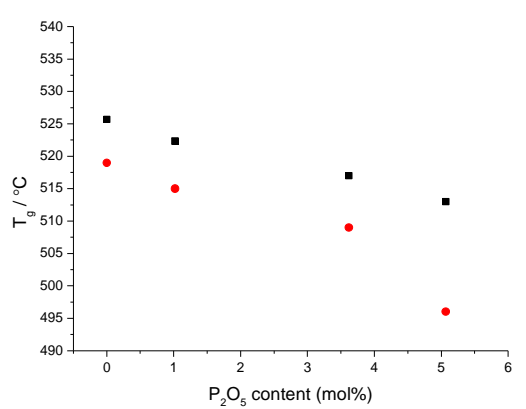


DENTMA-D-20-00341, R1

Fig 1. (a) XRD patterns of initial glasses. (b) Glass Transition Temperature ( $\mathrm{T}_{\mathrm{g}}$ ) against $\mathrm{P}_{2} \mathrm{O}_{5}$ content in the BGs ( $\mathbf{\square}$ : our data; $\bullet$ : data from O'Donnell [2018]). (c) FTIR spectra of BGs powders after $72 \mathrm{~h}$ immersion in $\alpha$-MEM.
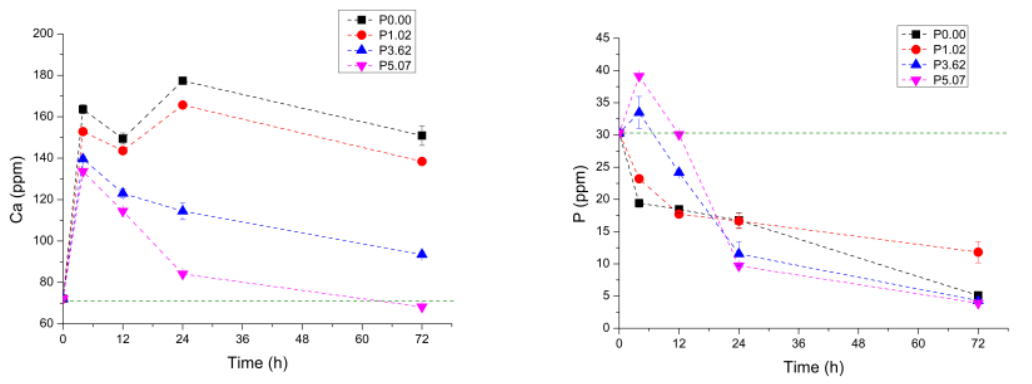

d

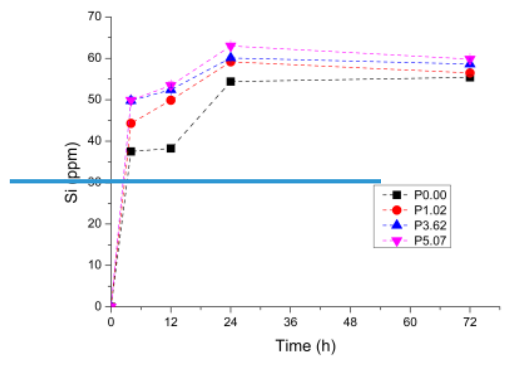


DENTMA-D-20-00341, R1
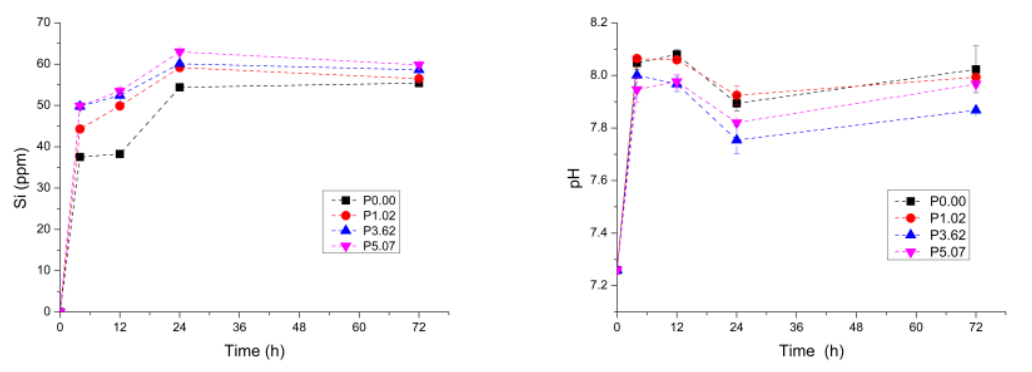

Fig 2. Measured ion concentration in BGs conditioned $\alpha$-MEM at 4, 12, 24 and 72h (a) calcium, (b) phosphorous and (c) silicon; (d) pH variation of BGs-conditioned culture $\alpha$-MEM media. Note that where error bars are not seen, they are smaller than the data point. The green dash line in (a) and (b) show $\mathrm{Ca}$ and $\mathrm{P}$ concentration in neat $\alpha$-MEM media respectively. 
DENTMA-D-20-00341, R1

A

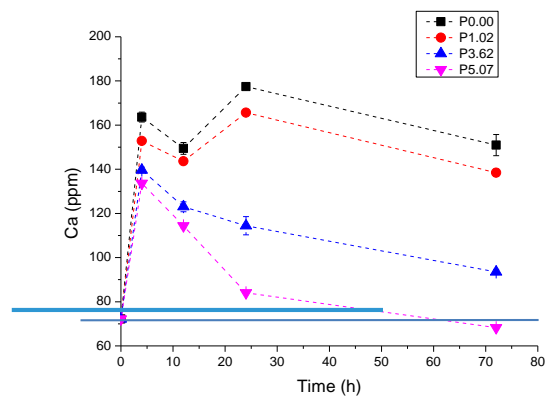

B

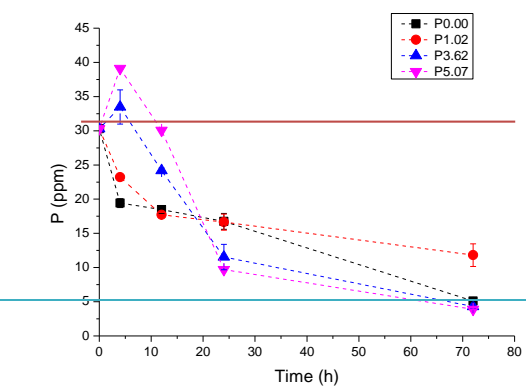

$-P 0.00$
$-P 1.02$
$-P 1.62$
$-P$
$-P 5.07$

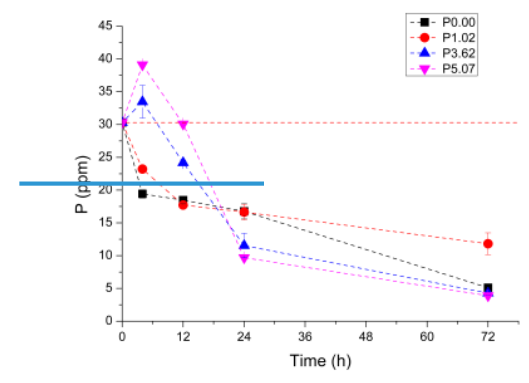

E

Đ 
DENTMA-D-20-00341, R1
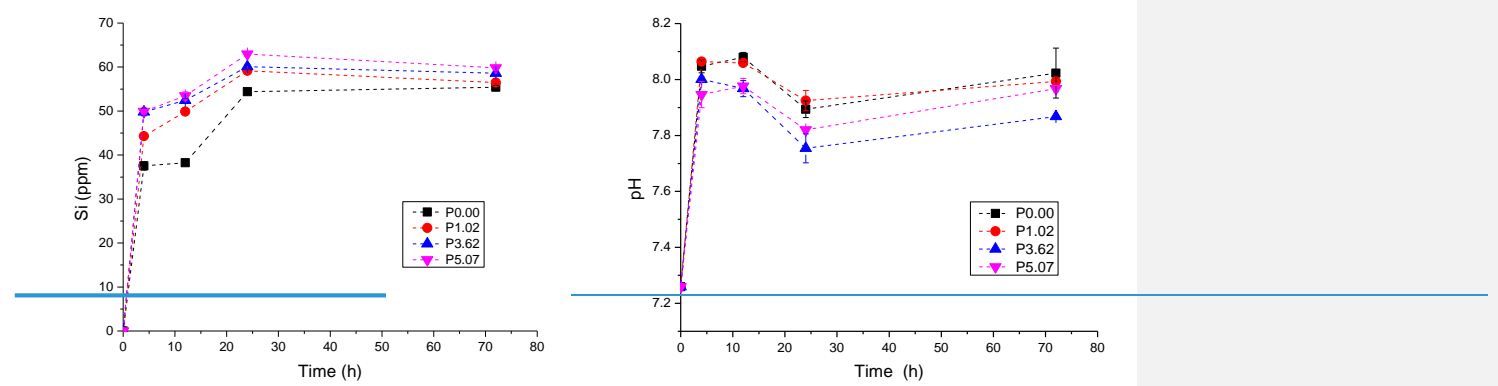

Fig 2. Measured ion concentration in BGs conditioned a-MEM at 4, 12, 24 and 72h (a) calcium, (b) phosphorous and (c) silicon; (d) pH variation of BGs conditionedculture $\alpha$-MEM media. Note that where error bars are not seen, they are smaller than the data point. AThe green dash line in (a) and (b) show $C a$ and $P$ concentration in neat $\alpha$ MEM media respectively. 
a

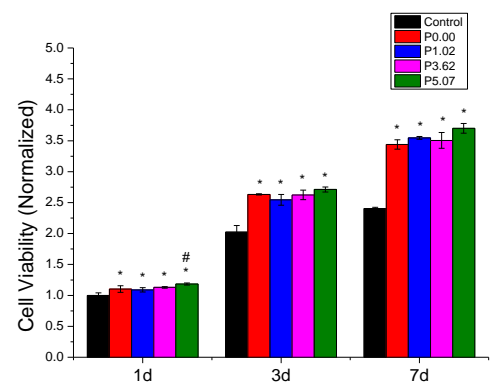

B

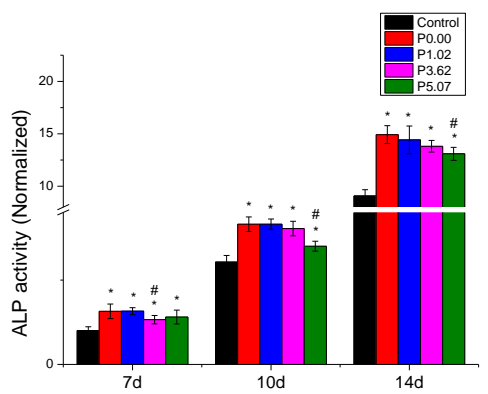

Fig 3. (a) Cell viability, (b) ALP activity of BG-conditioned media on MC3T3-E1. Data are presented as the mean \pm standard error mean $(n=6)$. $* P<0.05$, compared with the control group. \#P $<0.05$, compared with glass $\mathrm{P} 0.00$. 
DENTMA-D-20-00341, R1

a

B
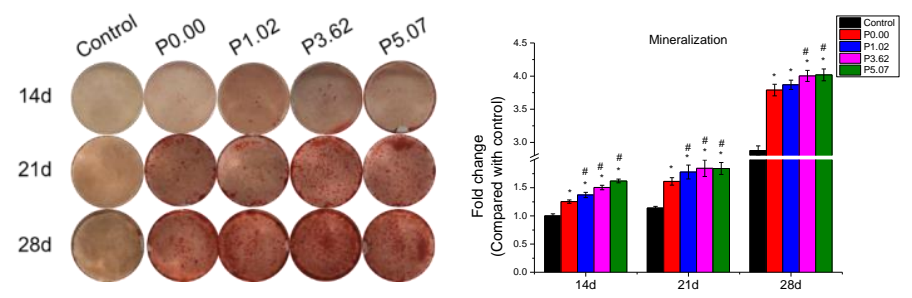

Fig 4. (a) Representative images of MC3T3-E1 mineralization presented by Alizarin Red S staining after BG-conditioned culture media treatment. (b) Quantification of Alizarin Red S in MC3T3-E1 expressed as a fold change compared to the relative group. Data were presented as mean $\pm \mathrm{SD}(\mathrm{n}=3)$. $* \mathrm{P}<0.05$, compared with the control. \#P $<$ 0.05 ,compared with glass P0.00. 
a

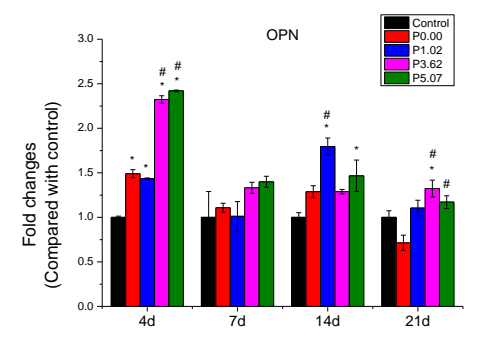

c

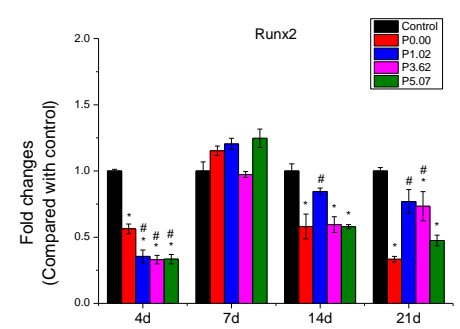

B

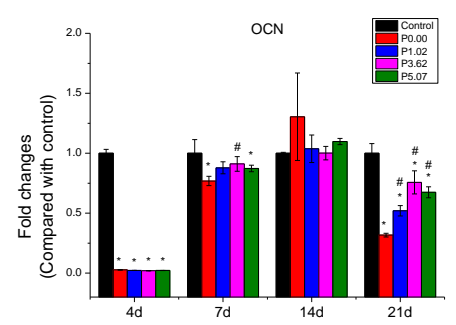

D

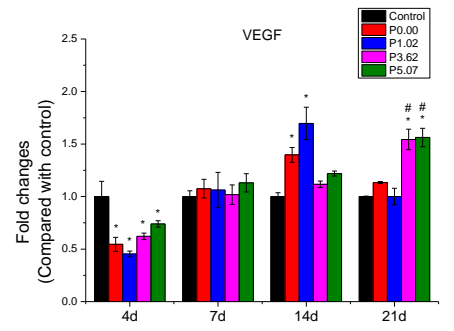

Fig 5. The expression of (a) OPN, (b) OCN, (c) RUNX2 and (d)VEGF in MC3T3-E1 following BGs-conditioned culture media treatment up to $21 \mathrm{~d}$ analyzed by RT-qPCR. Data were presented as the mean $\pm \mathrm{SD}(\mathrm{n}=3)$. $* \mathrm{P}<0.05$, compared with the control. \#P $<0.05$, compared with $\mathrm{P} 0.001$ group. Note that the genes expression of control group at different culturing datey wereas set as 1 and the expression level of the BGscontained culture media were normalized and compared against to-the control group atwith the same culturing days. 
DENTMA-D-20-00341, R1 
A

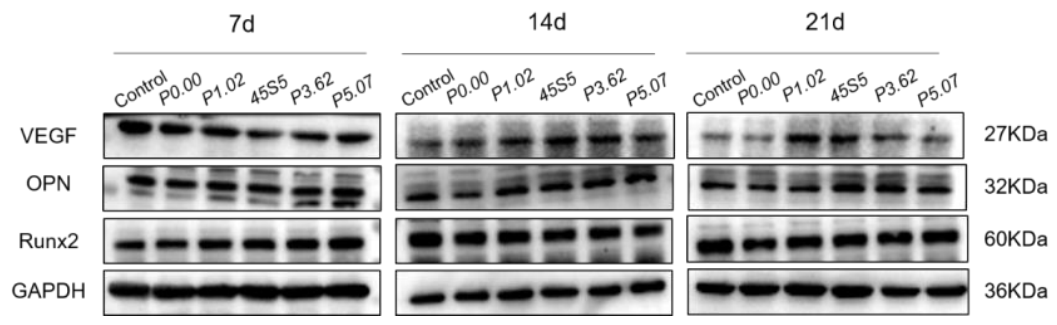

B

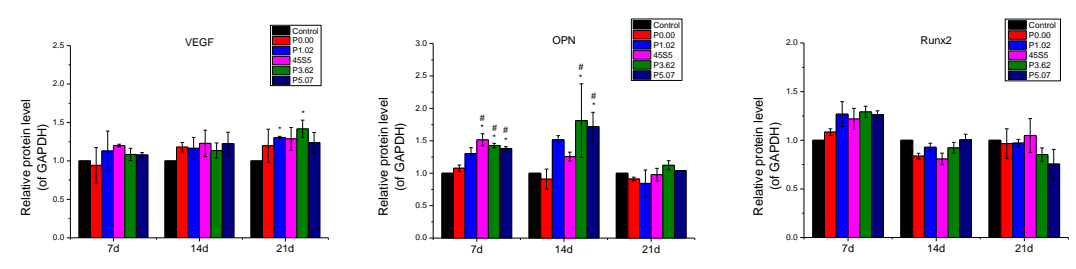

Fig 6. (a) Representative images of VEGF, OPN and RUNX2 protein expression analyzed by western blot. (b) Relative intensities of VEGF, OPN and RUNX2 in reference to GAPDH were showed as a fold change in comparison to the control group. Data were presented as the mean $\pm \mathrm{SD}$ from three independent experiments. ${ }^{*} \mathrm{P}<$ 0.05 ,compared with the control. \#P $<0.05$, compared with $\mathrm{P} 0.001$ group. Note that protein secretion level of control group at different culturing datey wereas set as 1 , while the protein concentration of the BGs groups was normalized and compared against the control with atthe same culturing days. 
$\mathbf{a}$

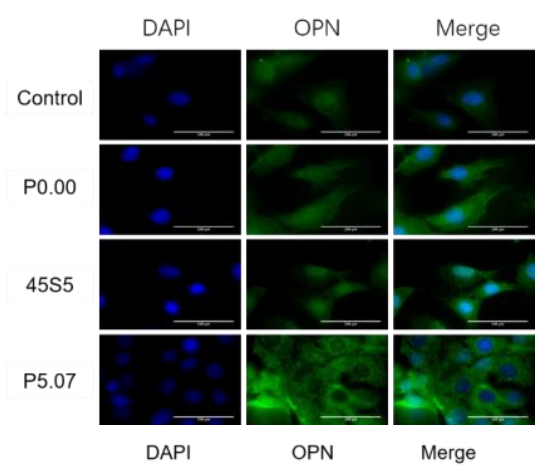

B

DAPI OPN Merge
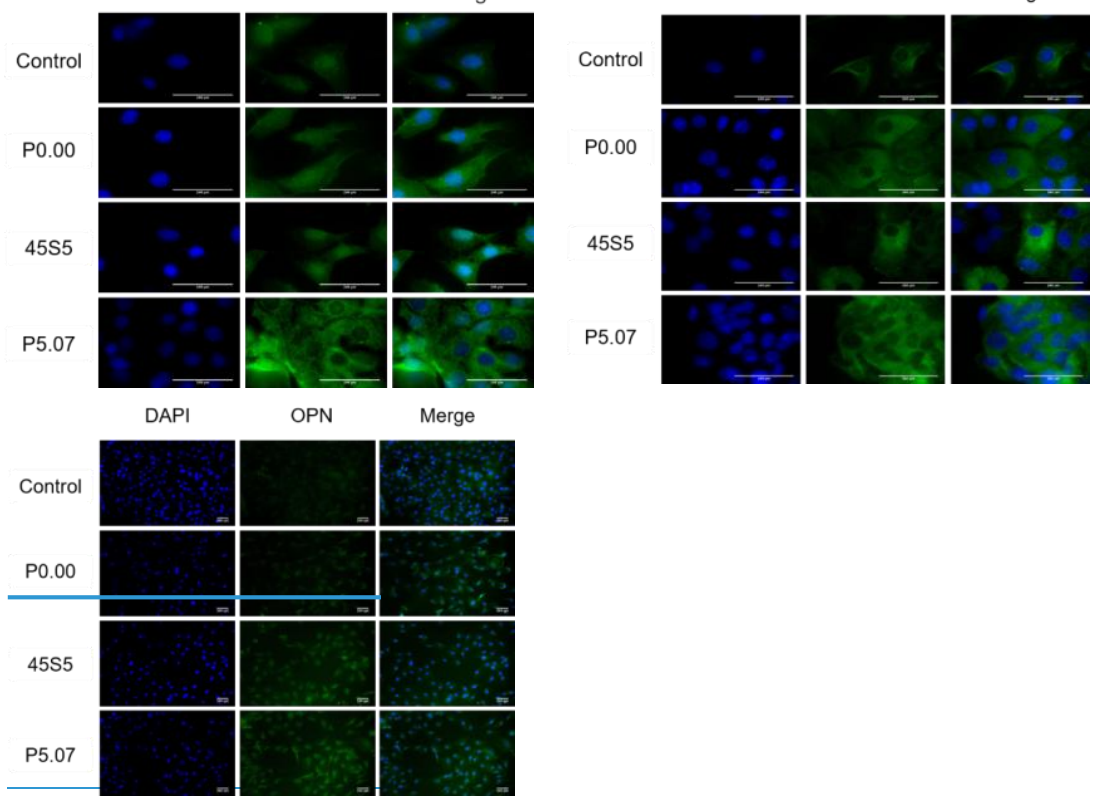

Fig 7. Representative fluorescence images show staining of DAPI (blue) and OPN (green) in MC3T3-E1 cells following BGs conditioned culture media treatment for (a) $7 \mathrm{~d}$ and (b) $14 \mathrm{~d}$. Scale bar $240 \mu \mathrm{m}$. The images in (a) and (b) were taken under low magnification and high magnification, respectively. 
DENTMA-D-20-00341, R1

a

Top view

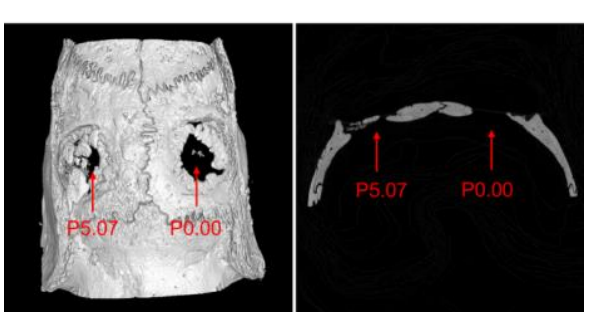

B

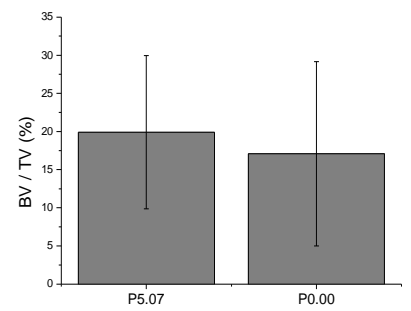

Fig 8. Micro-CT evaluation of bone regeneration in the rat calvarial defects implanted with the BGs at $8 \mathrm{w}$ post-surgery. (a) Top and sagittal sectional views of reconstructed images. (b) Bone volume/total volume (BV/TV) in the defects implanted with P5.07 and P0.00. Data were expressed as mean \pm SD from three individual rats. 


\section{Supplementary materials}

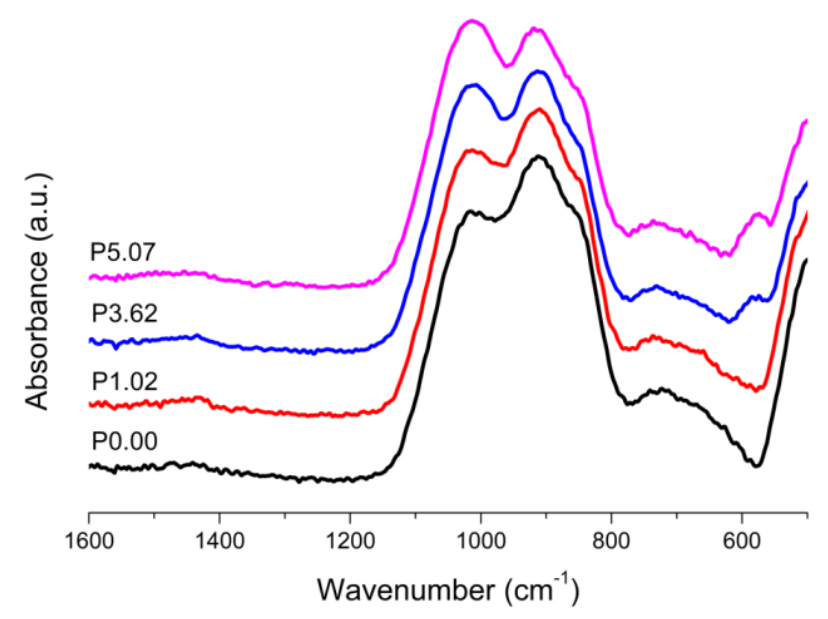

Fig S1. FTIR spectra of initial bioactive glass powders. 
Table S1: Sequences of primer pairs used for RT-qPCR analysis. Osteopontin (OPN), Osteocalcin (OCN), Runt-related transcription factor-2 (RUNX2), Vascular Endothelial Growth Factor (VEGF) are the genes of interest, while GAPDH is the reference gene [153].

\begin{tabular}{ll}
\hline Genes & Primer sequences \\
\hline OPN & Forward: GCAGTCTTCTGCGGCAGGCA \\
& Reverse: CACCGGGAGGGAGGAGGCAA \\
OCN & Forward: AGCAGCTTGGCCCAGACCTA \\
& Reverse: TAGCGCCGGAGTCTGTTCACTAC \\
RUNX2 & Forward: CACTGGCGGTGCAACAAGA \\
& Reverse: TTTCATAACAGCGGAGGCATTTC \\
VEGF & Forward: TTTCATAACAGCGGAGGCATTTC \\
& Reverse: TCTGCATGGTGATGTTGCTCTCTG \\
GAPDH & Forward: CTCCCACTCTTCCACCTTCG \\
& Reverse: TTGCTGTAGCCGTATTCATT \\
\hline
\end{tabular}

